\title{
Computational Singular Perturbation Analysis of Stochastic Chemical Systems with Stiffness ${ }^{\text {th }}$
}

\author{
Lijin Wang ${ }^{\mathrm{a}}$, Xiaoying Han ${ }^{\mathrm{b}, *}$, Yanzhao Cao ${ }^{\mathrm{b}}$, Habib N. Najm ${ }^{\mathrm{c}}$ \\ ${ }^{a}$ School of Mathematical Sciences, University of Chinese Academy of Sciences, \\ 19 YuQuan Road, Shijingshan District, Beijing 100049, China \\ b221 Parker Hall, Department of Mathematics and Statistics, \\ Auburn University, Auburn, AL 36849, USA \\ ${ }^{c}$ Sandia National Laboratories, P. O. Box 969, MS 9051 \\ Livermore, CA 94551, USA
}

\begin{abstract}
Computational singular perturbation (CSP) is a useful method for analysis, reduction, and time integration of stiff ordinary differential equation systems. It has found dominant utility, in particular, in chemical reaction systems with a large range of time scales at continuum and deterministic level. On the other hand, CSP is not directly applicable to chemical reaction systems at micro or meso-scale, where stochasticity plays an non-negligible role and thus has to be taken into account. In this work we develop a novel stochastic computational singular perturbation (SCSP) analysis and time integration framework, and associated algorithm, that can be used to not only construct accurately and efficiently the numerical solutions to stiff stochastic chemical reaction systems, but also analyze the dynamics of the reduced stochastic reaction systems. The algorithm is illustrated by an application to a benchmark stochastic differential equation model, and numerical experiments are carried out to demonstrate the effectiveness of the construction.
\end{abstract}

Keywords: Computational singular perturbation, model reduction, stochastic chemical reaction systems, chemical Langevin equation, stochastic Davis-Skodje model.

\footnotetext{
This work is partially supported by the National Science Foundation (grant number DMS1620027), National Natural Science Foundation of China (grant numbers 11471310 \& 11071251), and the U.S. Department of Energy, Office of Basic Energy Sciences, Division of Chemical Sciences, Geosciences and Biosciences. Sandia National Laboratories is a multi-program laboratory managed and operated by Sandia Corporation, a wholly owned subsidiary of Lockheed Martin Corporation, for the U.S. Department of Energy Nuclear Security Administration under contract DE-AC04-94-AL85000.

*Corresponding author

Email address: xzh0003eauburn. edu (Xiaoying Han)
} 


\section{Introduction}

Consider a well-stirred, thermally equilibrated mixture consisting of $N$ chemical species $\left\{S_{i}\right\}_{i=1, \ldots, N}$, which interact through $M$ chemical reaction channels

$$
\mathcal{R}_{j}: \sum_{i=1}^{N} a_{i j} S_{i} \longrightarrow \sum_{i=1}^{N} b_{i j} S_{i}, \quad j=1, \ldots, M,
$$

where $a_{i j}, b_{i j} \geq 0$ are the stoichiometric coefficients. Then $\gamma_{i j}:=b_{i j}-a_{i j}$ is the change in the population of $S_{i}$ caused by one $\mathcal{R}_{j}$ reaction, and $\gamma_{j}=\left(\gamma_{1 j}, \ldots, \gamma_{N j}\right)^{T}$ is the stoichiometric vector. Denote by $x_{i}(t)$ the concentration of species $S_{i}$ in the system at any time $t$, and $\boldsymbol{x}=\left(x_{1}, x_{2}, \ldots, x_{N}\right)^{T}$ the state vector. Then reaction rate equations (RREs) describing chemical kinetics at the continuum level are a system of ordinary differential equations (ODEs):

$$
\frac{\mathrm{d} \boldsymbol{x}}{\mathrm{d} t}=\sum_{j=1}^{M} \gamma_{j} R_{j}(\boldsymbol{x}):=\boldsymbol{f}(\boldsymbol{x}),
$$

where $R_{j}(\boldsymbol{x}), j=1, \ldots, M$, is the algebraic reaction rate of progress of the $j$ th reaction.

Detailed chemical reaction mechanisms typically involve large numbers of species and reactions, and exhibit wide ranges of time scales [16, 35, 47, 54, 64, 78]. Mathematically, a chemical reaction system at the continuum level, involving fast and slow time scales, results in a system of RREs of the form (1), with stiffness. Generally, stiff ODEs behave somewhat like differential-algebraic equations (DAEs), in the sense that their solution dynamics exhibit underlying algebraic manifolds. Fast time-scales are "exhausted" quickly, and solutions eventually move on a cascade of lower-dimensional, attracting, and invariant "slow manifolds" that characterize the long-term process dynamics. These manifolds are an expression of the partial-equilibration of fast processes, being characterized by algebraic relationships among a subset of the species. A differential "slow" sub-system is useful to approximate the slow evolution of the full system state when the system approaches this manifold. Analysis methods to identify these slow manifolds are subjects of intense study, as a means first and foremost for understanding underlying system dynamics $[23,33,38,50,51,56,75]$. Aside from this however, these manifolds can be used to either produce simplified/reduced chemical models with requisite dynamical accuracy [55, 71, 72], or as guideposts for projecting the ODEs onto the fast/slow subspaces, thereby providing means for fast explicit time integration of stiff ODE systems [73, 76].

There is a wide range of methods for analysis and reduction of chemical ODE systems. Reaction flux analysis methods rely on elimination of reactions with negligible rates of progress, with no attention to the dynamical landscape of the system, often in conjunction of graph models for the reaction network [44, 45]. The quasisteady-state approximation $[6,15,36,41,52,63,70,81]$, and the partial equilibrium approximation $[7,19,36,79]$ provide alternate means of examining the partial equilibrium of fast processes, without explicitly describing underlying manifolds. Both have 
been shown to have significant limitations [20]. Both intrinsic low-dimensional manifold (ILDM) [48], and rate-controlled constrained-equilibrium (RCCE) [34, 39, 58], methods deal explicitly with manifolds, although the former relies on an a priori fixed dimensionality of the manifold, while the latter requires a good deal of physical intuition to define the relevant constraints. In contrast, CSP is an automated computational procedure for analysis and discovery of low-dimensional manifolds, based on dynamical analysis of the underlying ODE system [12, 21-24, 28, 33, 36-38, 40, 46, 49$51,55,56,71-76]$.

CSP is a powerful analysis method for deterministic stiff ODE systems, providing a foundation for reliable model reduction and/or explicit time integration. However, chemical reactions are stochastic in principle, and the ODE system (1) is physically meaningful only when the system size is at continuum/macroscopic scales, where stochastic effects are averaged out. When particle counts are large, e.g., $\mathcal{O}\left(10^{3}\right)$, but not sufficiently so to make the continuum approximation viable, the chemical Langevin equations (CLEs), which are essentially the prototype of stochastic differential equations (SDEs), can be used to simulate the state dynamics [17].

For stochastic chemical reaction networks based on jump Markov processes, the quasi-steady-state approximation had been used to eliminate fast occurring reactions in $[8,26,57,59]$, and the reduction of the chemical master equation had been used in $[30,31,65,67]$. The reduction methods for stochastic chemical reaction networks governed by CLEs, however, are very limited to date. For SDEs with fast and slow time scales, the theory of stochastic geometric singular perturbation had been used to obtain the existence and an abstract descriptions of the random slow manifold in [4], and the theory of random dynamical system had been used to construct a geometric description of the random slow manifold in [29] while the noise is of a simplified structure. The extensive theory of random dynamical systems allows abstract analysis of stochastic stable manifolds (see, e.g., [1, 9, 13, 14, 42, 43, 60-62]), but is outside the scope of the present work. For linear Itô type of SDEs, model reduction methods analogous to balanced truncation in control theory were carried over in $[2,3,27,77$, 82]. Most recently a reduction method based on the CLE was developed in [80] to obtain a limit averaging system that approximates the slow reactions by averaging out the fast-reacting variables, and another method based on the CLE was developed in [10] to detect intrinsic slow variables by using anisotropic diffusion maps.

The main goal of this work is to develop computational algorithms analogous to the CSP, that can be used to numerically construct the random slow manifold and approximate the reduced dynamics on the manifold, for stochastic reaction systems governed by general fast-slow SDEs. In particular, we first generalize the key idea of deterministic CSP method to the stochastic context. The procedure is, however, nontrivial, as both the drift and the diffusion involve different time scales. We then develop an explicit time-scale splitting algorithm for stiff SDEs, that has an accuracy comparable to regular explicit stiff integrators for SDEs with small step lengths, but at a much lower cost. The rest of this paper is organized as follows. In Section 2 we develop the stochastic computational singular perturbation (SCSP) algorithm for stochastic fastslow SDEs. In Section 3 we apply the SCSP to bench mark stochastic Davis-Skodje system and present numerical experiments. To simplify the representation, some details of calculations performed in Section 3 are given in the appendix. Some closing 
remarks are given in Section 4.

\section{Stochastic computational singular perturbation (SCSP)}

Consider a stochastic reaction system of $N$ unknowns denoted by the column stochastic process vector $\boldsymbol{z}(t)=\left(z_{1}(t), z_{2}(t), \ldots, z_{N}(t)\right)^{T}$, with the governing stochastic differential equations (or chemical Langevin equations):

$$
\mathrm{d} \boldsymbol{z}=\boldsymbol{f}(\boldsymbol{z}) \mathrm{d} t+\boldsymbol{g}(\boldsymbol{z}) \circ \mathrm{d} W(t),
$$

where $\boldsymbol{f}$ and $\boldsymbol{g}$ are $N \times 1$ column vectors, $W(t) \in \mathbb{R}$ is a standard Brownian motion, and $\circ$ denotes the Stratonovich product. Our goal is to find computationally another (better) representation than the physical representation (2), such that the fast dynamics and the slow dynamics can be separated.

Remark 2.1. We start with a single Brownian motion here for clarity of exposition, but the algorithm to be developed can be extended to the case where $\boldsymbol{g}=\left(g_{i j}\right)_{i=1 . . N, j=1 . . M}$ is an $N \times M$ matrix and $W(t)=\left(W_{1}(t), \ldots, W_{M}(t)\right)^{T}$ is an $M$ dimensional Brownian motion, by writing

$$
\boldsymbol{g}(\boldsymbol{z}) \circ d W(t)=\sum_{j=1}^{M} g_{j}(\boldsymbol{z}) \circ d W_{j}(t),
$$

where $g_{j}(\boldsymbol{z})=\left(g_{1 j}, \ldots, g_{N j}\right)^{T}$ is the $j$-th column of $\boldsymbol{g}(\boldsymbol{z})$.

Similar to CSP, the stochastic computational singular perturbation (SCSP) aims at splitting the amplitudes of both $\boldsymbol{f}$ and $\boldsymbol{g}$ in (2) into two classes, rapid and slow, as

$$
\boldsymbol{f}=\left(\begin{array}{c}
\boldsymbol{f}^{r} \\
\boldsymbol{f}^{s}
\end{array}\right), \quad \boldsymbol{g}=\left(\begin{array}{c}
\boldsymbol{g}^{r} \\
\boldsymbol{g}^{s}
\end{array}\right),
$$

where $\boldsymbol{f}^{r}=\left(f_{1}, \ldots, f_{m}\right)^{T}$ and $\boldsymbol{g}^{r}=\left(g_{1}, \ldots, g_{m}\right)^{T}$ are $m$ vectors representing the deterministic and stochastic fast amplitudes respectively, and $\boldsymbol{f}^{s}=\left(f_{m+1}, \ldots, f_{N}\right)^{T}$ and $\boldsymbol{g}^{s}=\left(g_{m+1}, \ldots, g_{N}\right)^{T}$ are $N-m$ vectors representing the deterministic and stochastic slow amplitudes respectively.

Given any two sets of basis vectors, $\boldsymbol{a}_{i}$ and $\boldsymbol{\alpha}_{i}$ for $i=1, \ldots, N$, let $\boldsymbol{b}_{i}$ and $\boldsymbol{\beta}_{i}$ denote the corresponding inverse row vectors of $\boldsymbol{a}_{i}$ and $\boldsymbol{\alpha}_{i}$, respectively, satisfying

$$
\boldsymbol{b}_{i} \cdot \boldsymbol{a}_{j}=\boldsymbol{\beta}_{i} \cdot \boldsymbol{\alpha}_{j}=\delta_{i j}, \quad i, j=1, \cdots, N .
$$

Denote by $A:=\left[\boldsymbol{a}_{1} \ldots \boldsymbol{a}_{N}\right]$ and $\mathcal{A}:=\left[\boldsymbol{\alpha}_{1} \ldots \boldsymbol{\alpha}_{N}\right]$, then the splitting (3) suggests the decompositions of $A=\left[A^{r} A^{s}\right]$ and $\mathcal{A}=\left[\mathcal{A}^{r} \mathcal{A}^{s}\right]$ where

$$
\begin{array}{ll}
A^{r}=\left[\boldsymbol{a}_{1} \ldots \boldsymbol{a}_{m}\right] \in \mathbb{R}_{N \times m}, & A^{s}=\left[\boldsymbol{a}_{m+1} \ldots \boldsymbol{a}_{N}\right] \in \mathbb{R}_{N \times(N-m)}, \\
\mathcal{A}^{r}=\left[\boldsymbol{\alpha}_{1} \ldots \boldsymbol{\alpha}_{m}\right] \in \mathbb{R}_{N \times m}, & \mathcal{A}^{s}=\left[\boldsymbol{\alpha}_{m+1} \ldots \boldsymbol{\alpha}_{N}\right] \in \mathbb{R}_{N \times(N-m)} .
\end{array}
$$


Correspondingly, the matrices $B:=\left[\boldsymbol{b}_{1}^{T} \ldots \boldsymbol{b}_{N}^{T}\right]^{T}$ and $\mathcal{B}:=\left[\boldsymbol{\beta}_{1}^{T} \ldots \boldsymbol{\beta}_{N}^{T}\right]^{T}$ can be decomposed as $B=\left[\left[B^{r}\right]^{T}\left[B^{s}\right]^{T}\right]^{T}$ and $\mathcal{B}=\left[\left[\mathcal{B}^{r}\right]^{T}\left[\mathcal{B}^{s}\right]^{T}\right]^{T}$, where

$$
\begin{array}{ll}
B^{r}=\left[\boldsymbol{b}_{1}^{T} \ldots \boldsymbol{b}_{m}^{T}\right]^{T} \in \mathbb{R}_{m \times N}, & B^{s}=\left[\boldsymbol{b}_{m+1}^{T} \ldots \boldsymbol{b}_{N}^{T}\right]^{T} \in \mathbb{R}_{(N-m) \times N}, \\
\mathcal{B}^{r}=\left[\boldsymbol{\beta}_{1}^{T} \ldots \boldsymbol{\beta}_{m}^{T}\right]^{T} \in \mathbb{R}_{m \times N}, & \mathcal{B}^{s}=\left[\boldsymbol{\beta}_{m+1}^{T} \ldots \boldsymbol{\beta}_{N}^{T}\right]^{T} \in \mathbb{R}_{(N-m) \times N} .
\end{array}
$$

Moreover, the orthogonality conditions (4) between $\boldsymbol{a}_{i}$ and $\boldsymbol{b}_{i}$ and $\boldsymbol{\alpha}_{i}$ and $\boldsymbol{\beta}_{i}$ imply that

$$
\begin{array}{llll}
B^{r} A^{r}=I_{m}, & B^{s} A^{r}=0, & B^{r} A^{s}=0, & B^{s} A^{s}=I_{N-m}, \\
\mathcal{B}^{r} \mathcal{A}^{r}=I_{m}, & \mathcal{B}^{s} \mathcal{A}^{r}=0, & \mathcal{B}^{r} \mathcal{A}^{s}=0, & \mathcal{B}^{s} \mathcal{A}^{s}=I_{N-m}, \\
A^{r} B^{r}+A^{s} B^{s}=I_{N}, & \mathcal{A}^{r} \mathcal{B}^{r}+\mathcal{A}^{s} \mathcal{B}^{s}=I_{N} .
\end{array}
$$

The deterministic and stochastic vector field of (2) can then be written as

$$
\boldsymbol{f}(\boldsymbol{z})=A^{r} \boldsymbol{f}^{r}+A^{s} \boldsymbol{f}^{s}, \quad \boldsymbol{g}(\boldsymbol{z})=\mathcal{A}^{r} \boldsymbol{g}^{r}+\mathcal{A}^{s} \boldsymbol{g}^{s},
$$

where

$$
\boldsymbol{f}^{r}=B^{r} \cdot \boldsymbol{f}(\boldsymbol{z}), \quad \boldsymbol{f}^{s}=B^{s} \cdot \boldsymbol{f}(\boldsymbol{z}), \quad \boldsymbol{g}^{r}=\mathcal{B}^{r} \cdot \boldsymbol{g}(\boldsymbol{z}), \quad \boldsymbol{g}^{s}=\mathcal{B}^{s} \cdot \boldsymbol{g}(\boldsymbol{z}) .
$$

Note that the fast and slow amplitudes $\boldsymbol{f}^{r}, \boldsymbol{f}^{s}, \boldsymbol{g}^{r}, \boldsymbol{g}^{s}$ evolve with respect to time. Denoting by $J_{\boldsymbol{f}}$ the Jacobian matrix of $\boldsymbol{f}$ and differentiating the first equation of (6) along solutions to the system (2) gives

$$
\begin{aligned}
\mathrm{d} \boldsymbol{f}^{r} & =\mathrm{d} B^{r} \boldsymbol{f}(\boldsymbol{z})+B^{r} J_{\boldsymbol{f}} \mathrm{d} \boldsymbol{z} \\
& =\mathrm{d} B^{r}\left(A^{r} \boldsymbol{f}^{r}+A^{s} \boldsymbol{f}^{s}\right)+\left(\Lambda^{r r} \boldsymbol{f}^{r}+\Lambda^{r s} \boldsymbol{f}^{s}\right) \mathrm{d} t+\left(V^{r r} \boldsymbol{g}^{r}+V^{r s} \boldsymbol{g}^{s}\right) \circ \mathrm{d} W(t),
\end{aligned}
$$

where

$$
\Lambda^{r r}=B^{r} J_{\boldsymbol{f}} A^{r}, \quad \Lambda^{r s}=B^{r} J_{\boldsymbol{f}} A^{s}, \quad V^{r r}=B^{r} J_{\boldsymbol{f}} \mathcal{A}^{r}, \quad V^{r s}=B^{r} J_{\boldsymbol{f}} \mathcal{A}^{s} .
$$

Similarly, denoting by $J_{g}$ the Jacobian matrix of $g$ and differentiating the second, third and fourth equation in (6) along solutions to the system (2) gives

$$
\begin{aligned}
\mathrm{d} \boldsymbol{f}^{s} & =\mathrm{d} B^{s}\left(A^{r} \boldsymbol{f}^{r}+A^{s} \boldsymbol{f}^{s}\right)+\left(\Lambda^{s r} \boldsymbol{f}^{r}+\Lambda^{s s} \boldsymbol{f}^{s}\right) \mathrm{d} t+\left(V^{s r} \boldsymbol{g}^{r}+V^{s s} \boldsymbol{g}^{s}\right) \circ \mathrm{d} W(t), \\
\mathrm{d} \boldsymbol{g}^{r} & =\mathrm{d} \mathcal{B}^{r}\left(\mathcal{A}^{r} \boldsymbol{g}^{r}+\mathcal{A}^{s} \boldsymbol{g}^{s}\right)+\left(\Gamma^{r r} \boldsymbol{f}^{r}+\Gamma^{r s} \boldsymbol{f}^{s}\right) \mathrm{d} t+\left(T^{r r} \boldsymbol{g}^{r}+T^{r s} \boldsymbol{g}^{s}\right) \circ \mathrm{d} W(t), \\
\mathrm{d} \boldsymbol{g}^{s} & =\mathrm{d} \mathcal{B}^{s}\left(\mathcal{A}^{r} \boldsymbol{g}^{r}+\mathcal{A}^{s} \boldsymbol{g}^{s}\right)+\left(\Gamma^{s r} \boldsymbol{f}^{r}+\Gamma^{s s} \boldsymbol{f}^{s}\right) \mathrm{d} t+\left(T^{s r} \boldsymbol{g}^{r}+T^{s s} \boldsymbol{g}^{s}\right) \circ \mathrm{d} W(t),
\end{aligned}
$$

where

$$
\begin{aligned}
& \Lambda^{s r}=B^{s} J_{f} A^{r}, \quad \Lambda^{s s}=B^{s} J_{\boldsymbol{f}} A^{s}, \quad V^{s r}=B_{r}^{s} J_{\boldsymbol{f}} \mathcal{A}^{r}, \quad V^{s s}=B^{s} J_{\boldsymbol{f}} \mathcal{A}^{s}, \\
& \Gamma^{r r}=\mathcal{B}^{r} J_{\boldsymbol{g}} A^{r}, \quad \Gamma^{r s}=\mathcal{B}^{r} J_{\boldsymbol{g}} A^{s}, \quad T^{r r}=\mathcal{B}^{r} J_{\boldsymbol{g}} \mathcal{A}^{r}, \quad T^{r s}=\mathcal{B}^{r} J_{\boldsymbol{g}} \mathcal{A}^{s}, \\
& \Gamma^{s r}=\mathcal{B}^{s} J_{\boldsymbol{g}} A^{r}, \quad \Gamma^{s s}=\mathcal{B}^{s} J_{\boldsymbol{g}} A^{s}, \quad T^{s r}=\mathcal{B}^{s} J_{\boldsymbol{g}} \mathcal{A}^{r}, \quad T^{s s}=\mathcal{B}^{s} J_{\boldsymbol{g}} \mathcal{A}^{s} .
\end{aligned}
$$


Denote by

$$
\mathrm{d} \omega(t)=\operatorname{Diag}\left[I_{m} \mathrm{~d} t, I_{N-m} \mathrm{~d} t, I_{m} \circ \mathrm{d} W(t), I_{N-m} \circ \mathrm{d} W(t)\right] .
$$

Note that the notation (11) is non-conventional; it is introduced to avoid over complicated formulas in the following derivations. Start from any set of basis, $A^{r}, A^{s}, \mathcal{A}^{r}$, $\mathcal{A}^{s}$ with corresponding inverse basis $B^{r}, B^{s}, \mathcal{B}^{r}, \mathcal{B}^{s}$ and amplitudes of modes $\boldsymbol{f}^{r}, \boldsymbol{f}^{s}$, $\boldsymbol{g}^{r}, \boldsymbol{g}^{s}$, by using (7) - (10) we obtain the time evolution of all modes

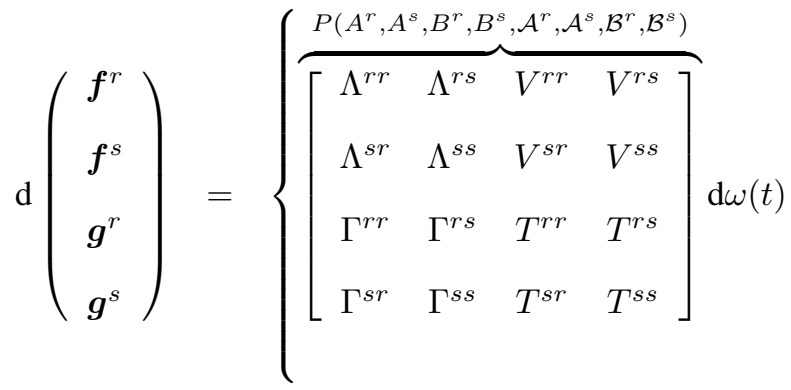

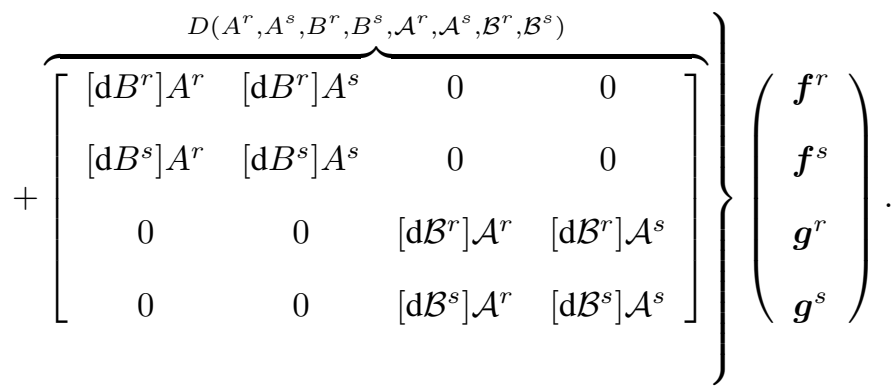

It can be seen from (12) that when the off block-diagonal entries of $P$ or $D$, i.e., $\Lambda^{r s}$, $\Lambda^{s r}, V^{r s}, V^{s r}, \Gamma^{r s}, \Gamma^{s r}, T^{r s}, T^{s r},\left[\mathrm{~d} B^{r}\right] A^{s},\left[\mathrm{~d} B^{s}\right] A^{r},\left[\mathrm{~d} \mathcal{B}^{r}\right] \mathcal{A}^{s},\left[\mathrm{~d} \mathcal{B}^{s}\right] \mathcal{A}^{r}$, are nontrivial, the evolutions of $\boldsymbol{f}^{r}$ and $\boldsymbol{g}^{r}$ are coupled with the evolutions of $\boldsymbol{f}^{s}$ and $\boldsymbol{g}^{s}$. Similar to CSP, a refinement procedure can be designed to reduce iteratively the magnitudes of off block-diagonal entries and hence separate the evolution of $\boldsymbol{f}^{r}$ and $\boldsymbol{g}^{r}$ from $\boldsymbol{f}^{s}$ and $\boldsymbol{g}^{s}$.

Notice that the evolution system (12) can be simplified by eliminating the $D$ matrix. This can be done by starting from a constant initial trial set of time-independent basis, $A_{0}^{r}, A_{0}^{s}, \mathcal{A}_{0}^{r}, \mathcal{A}_{0}^{s}$ (e.g., the eigenvectors of $J_{\boldsymbol{f}}$ and $J_{\boldsymbol{g}}$ ) with corresponding inverses $B_{0}^{r}$, $B_{0}^{s}, \mathcal{B}_{0}^{r}, \mathcal{B}_{0}^{s}$, that give $D\left(A_{0}^{r}, A_{0}^{s}, B_{0}^{r}, B_{0}^{s}, \mathcal{A}_{0}^{r}, \mathcal{A}_{0}^{s}, \mathcal{B}_{0}^{r}, \mathcal{B}_{0}^{s}\right)=0$. Denote by $A_{k}^{r}, A_{k}^{s}$, $\mathcal{A}_{k}^{r}, \mathcal{A}_{k}^{s}$ the $k$-th refined basis vectors obtained after $k$ iterations of refinements and by $B_{k}^{r}, B_{k}^{s}, \mathcal{B}_{k}^{r}, \mathcal{B}_{k}^{s}$ their corresponding inverses. Let $\boldsymbol{f}_{(k)}^{r}, \boldsymbol{f}_{(k)}^{s}, \boldsymbol{g}_{(k)}^{r}$ and $\boldsymbol{g}_{(k)}^{s}$ be the magnitude of each mode projected on the $k$-th refined basis. To simplify notations, denote by

$$
\ell_{k}:=\left(\boldsymbol{f}_{(k)}^{r}, \boldsymbol{f}_{(k)}^{s}, \boldsymbol{g}_{(k)}^{r}, \boldsymbol{g}_{(k)}^{s}\right)^{T} .
$$

Then the evolution of $\ell_{0}$ follows

$$
\mathrm{d} \ell_{0}=P\left(A_{0}^{r}, A_{0}^{s}, B_{0}^{r}, B_{0}^{s}, \mathcal{A}_{0}^{r}, \mathcal{A}_{0}^{s}, \mathcal{B}_{0}^{r}, \mathcal{B}_{0}^{s}\right) \mathrm{d} \omega(t) \ell_{0}:=P_{0} \mathrm{~d} \omega(t) \ell_{0} .
$$


To derive the iterative formulation for basis refinements, we start from (13) and assume that the evolution of $\ell_{k}$ follows

$$
\mathrm{d} \ell_{k}=P_{k} \mathrm{~d} \omega(t) \ell_{k}, \quad P_{k}=\left[\begin{array}{cccc}
\Lambda_{k}^{r r} & \Lambda_{k}^{r s} & V_{k}^{r r} & V_{k}^{r s} \\
\Lambda_{k}^{s r} & \Lambda_{k}^{s s} & V_{k}^{s r} & V_{k}^{s s} \\
\Gamma_{k}^{r r} & \Gamma_{k}^{r s} & T_{k}^{r r} & T_{k}^{r s} \\
\Gamma_{k}^{s r} & \Gamma_{k}^{s s} & T_{k}^{s r} & T_{k}^{s s}
\end{array}\right] .
$$

Each iteration of basis refinements consists of two steps of refinements.

\subsection{Basis refinement Step I}

This step depresses the magnitudes of $\Lambda_{k}^{r s}$ and $V_{k}^{r s}$ and thus weakens the coupling of fast modes from the slow. To this end, let

$$
Q_{k}=\left[\begin{array}{cccc}
I_{m} & -\left[\Lambda_{k}^{r r}\right]^{-1} \Lambda_{k}^{r s} & 0 & 0 \\
0 & I_{N-m} & 0 & 0 \\
0 & 0 & I_{m} & -\left[V_{k}^{r r}\right]^{-1} V_{k}^{r s} \\
0 & 0 & 0 & I_{N-m}
\end{array}\right],
$$

and update $\ell_{k}$ to $\tilde{\ell}_{k}=Q_{k}^{-1} \ell_{k}$, i.e., $\tilde{\ell}_{k}=\left(\left[\boldsymbol{f}_{(k+1)}^{r}\right]^{T},\left[\boldsymbol{f}_{(k)}^{s}\right]^{T},\left[\boldsymbol{g}_{(k+1)}^{r}\right]^{T},\left[\boldsymbol{g}_{(k)}^{s}\right]^{T}\right)$ where

$$
\boldsymbol{f}_{(k+1)}^{r}=\boldsymbol{f}_{(k)}^{r}+\left[\Lambda_{k}^{r r}\right]^{-1} \Lambda_{k}^{r s} \boldsymbol{f}_{(k)}^{s}, \quad \boldsymbol{g}_{(k+1)}^{r}=\boldsymbol{g}_{(k)}^{r}+\left[V_{k}^{r r}\right]^{-1} V_{k}^{r s} \boldsymbol{g}_{(k)}^{s} .
$$

Accordingly the basis vectors can be updated by

$$
\begin{array}{rlrl}
B_{k+1}^{r} & =B_{k}^{r}+\left[\Lambda_{k}^{r r}\right]^{-1} \Lambda_{k}^{r s} B_{k}^{s}, & & \mathcal{B}_{k+1}^{r}=\mathcal{B}_{k}^{r}+\left[V_{k}^{r r}\right]^{-1} V_{k}^{r s} \mathcal{B}_{k}^{s}, \\
A_{k+1}^{s}=\left[I_{N}-A_{k}^{r} B_{k+1}^{r}\right] A_{k}^{s}, & & \mathcal{A}_{k+1}^{s}=\left[I_{N}-\mathcal{A}_{k}^{r} \mathcal{B}_{k+1}^{r}\right] \mathcal{A}_{k}^{s} .
\end{array}
$$

Moreover, by using (14) and (15) the evolution of $\boldsymbol{f}_{(k+1)}^{r}$ can be calculated to be

$$
\begin{aligned}
\mathrm{d} \boldsymbol{f}_{(k+1)}^{r} & =\mathrm{d} \boldsymbol{f}_{(k)}^{r}+\mathrm{d}\left(\left[\Lambda_{k}^{r r}\right]^{-1} \Lambda_{k}^{r s}\right) \boldsymbol{f}_{(k)}^{s}+\left[\Lambda_{k}^{r r}\right]^{-1} \Lambda_{k}^{r s} \mathrm{~d} \boldsymbol{f}_{(k)}^{s} \\
& =\left(\tilde{\Lambda}_{k}^{r r} \boldsymbol{f}_{(k+1)}^{r}+\tilde{\Lambda}_{k}^{r s} \boldsymbol{f}_{(k)}^{s}\right) \mathrm{d} t+\left(\tilde{V}_{k}^{r r} \boldsymbol{g}_{(k+1)}^{r}+\tilde{V}_{k}^{r s} \boldsymbol{g}_{(k)}^{s}\right) \circ \mathrm{d} W(t),
\end{aligned}
$$

where

$$
\begin{aligned}
\tilde{\Lambda}_{k}^{r r} & =\Lambda_{k}^{r r}+\left[\Lambda_{k}^{r r}\right]^{-1} \Lambda_{k}^{r s} \Lambda_{k}^{s r}, \\
\tilde{\Lambda}_{k}^{r s} & =\frac{\mathrm{d}}{\mathrm{d} t}\left(\left[\Lambda_{k}^{r r}\right]^{-1} \Lambda_{k}^{r s}\right)+\left[\Lambda_{k}^{r r}\right]^{-1} \Lambda_{k}^{r s}\left(\Lambda_{k}^{s s}-\Lambda_{k}^{s r}\left[\Lambda_{k}^{r r}\right]^{-1} \Lambda_{k}^{r s}\right), \\
\tilde{V}_{k}^{r r} & =V_{k}^{r r}+\left[\Lambda_{k}^{r r}\right]^{-1} \Lambda_{k}^{r s} V_{k}^{s r}, \\
\tilde{V}_{k}^{r s} & =\left[\Lambda_{k}^{r r}\right]^{-1} \Lambda_{k}^{r s}\left(V_{k}^{s s}-V_{k}^{s r}\left[V_{k}^{r r}\right]^{-1} V_{k}^{r s}\right) .
\end{aligned}
$$


Similarly the evolution of $\boldsymbol{g}_{k+1}^{r}$ can be calculated by (14) and (15) to be

$$
\begin{aligned}
\mathrm{d} \boldsymbol{g}_{(k+1)}^{r} & =\mathrm{d} \boldsymbol{g}_{(k)}^{r}+\mathrm{d}\left(\left[V_{k}^{r r}\right]^{-1} V_{k}^{r s}\right) \boldsymbol{g}_{(k)}^{s}+\left[V_{k}^{r r}\right]^{-1} V_{k}^{r s} \mathrm{~d} \boldsymbol{g}_{(k)}^{s} \\
& =\left(\tilde{\Gamma}_{k}^{r r} \boldsymbol{f}_{(k+1)}^{r}+\tilde{\Gamma}_{k}^{r s} \boldsymbol{f}_{(k)}^{s}\right) \mathrm{d} t+\left(\tilde{T}_{k}^{r r} \boldsymbol{g}_{(k+1)}^{r}+\tilde{T}_{k}^{r s} \boldsymbol{g}_{(k)}^{s}\right) \circ \mathrm{d} W(t),
\end{aligned}
$$

where

$$
\begin{aligned}
\tilde{\Gamma}_{k}^{r r}= & \Gamma_{k}^{r r}+\left[V_{k}^{r r}\right]^{-1} V_{k}^{r s} \Gamma_{k}^{s r}, \\
\tilde{\Gamma}_{k}^{r s}= & \Gamma_{k}^{r s}-\Gamma_{k}^{r r}\left[\Lambda_{k}^{r r}\right]^{-1} \Lambda^{r s}+\left[V_{k}^{r r}\right]^{-1} V_{k}^{r s}\left(\Gamma_{k}^{s s}-\Gamma_{k}^{s r}\left[\Lambda_{k}^{r r}\right]^{-1} \Lambda_{k}^{r s}\right), \\
\tilde{T}_{k}^{r r}= & T_{k}^{r r}+\left[V_{k}^{r r}\right]^{-1} V_{k}^{r s} T_{k}^{s r}, \\
\tilde{T}_{k}^{r s}= & \frac{\mathrm{d}}{\mathrm{d} W(t)}\left(\left[V_{k}^{r r}\right]^{-1} V_{k}^{r s}\right)+T_{k}^{r s}-T_{k}^{r r}\left[V_{k}^{r r}\right]^{-1} V_{k}^{r s} \\
\quad & \quad+\left[V_{k}^{r r}\right]^{-1} V_{k}^{r s}\left(T_{k}^{s s}-T_{k}^{s r}\left[V_{k}^{r r}\right]^{-1} V_{k}^{r s}\right) .
\end{aligned}
$$

Remark 2.2. The notation $\frac{d}{d W(t)}$ here and in the sequel is non-conventional; it is only a formal notation for the sake of simplicity. All such terms in the refinement procedure appear in a matrix multiplied by $d \omega(t)$ that cancels out the $d W(t)$ in the denominator.

In addition, the evolutions of $\boldsymbol{f}_{(k)}^{s}$ and $\boldsymbol{g}_{(k)}^{s}$ can be expressed as

$$
\begin{aligned}
& \mathrm{d} \boldsymbol{f}_{(k)}^{s}=\left(\Lambda_{k}^{s r} \boldsymbol{f}_{(k+1)}^{r}+\tilde{\Lambda}_{k}^{s s} \boldsymbol{f}_{(k)}^{s}\right) \mathrm{d} t+\left(V_{k}^{s r} \boldsymbol{g}_{(k+1)}^{r}+\tilde{V}_{k}^{s s} \boldsymbol{g}_{(k)}^{s}\right) \circ \mathrm{d} W(t), \\
& \mathrm{d} \boldsymbol{g}_{(k)}^{s}=\left(\Gamma_{k}^{s r} \boldsymbol{f}_{(k+1)}^{r}+\tilde{\Gamma}_{k}^{s s} \boldsymbol{f}_{(k)}^{s}\right) \mathrm{d} t+\left(T_{k}^{s r} \boldsymbol{g}_{(k+1)}^{r}+\tilde{T}_{k}^{s s} \boldsymbol{g}_{(k)}^{s}\right) \circ \mathrm{d} W(t),
\end{aligned}
$$

where

$$
\begin{aligned}
& \tilde{\Lambda}_{k}^{s s}=\Lambda_{k}^{s s}-\Lambda_{k}^{s r}\left[\Lambda_{k}^{r r}\right]^{-1} \Lambda_{k}^{r s}, \quad \tilde{V}_{k}^{s s}=V_{k}^{s s}-V_{k}^{s r}\left[V_{k}^{r r}\right]^{-1} V_{k}^{r s} \\
& \tilde{\Gamma}_{k}^{s s}=\Gamma_{k}^{s s}-\Gamma_{k}^{s r}\left[\Lambda_{k}^{r r}\right]^{-1} \Lambda_{k}^{r s}, \quad \tilde{T}_{k}^{s s}=T_{k}^{s s}-T_{k}^{s r}\left[V_{k}^{r r}\right]^{-1} V_{k}^{r s} .
\end{aligned}
$$

In summary after the first refinement we obtain

$$
\mathrm{d} \tilde{\ell}_{k}=\tilde{P}_{k} \mathrm{~d} \omega(t) \tilde{\ell}_{k}
$$

where

$$
\tilde{P}_{k} \mathrm{~d} \omega(t)=\left[\begin{array}{cccc}
\tilde{\Lambda}_{k}^{r r} & \tilde{\Lambda}_{k}^{r s} & \tilde{V}_{k}^{r r} & \tilde{V}_{k}^{r s} \\
\Lambda_{k}^{s r} & \tilde{\Lambda}_{k}^{s s} & V_{k}^{s r} & \tilde{V}_{k}^{s s} \\
\tilde{\Gamma}_{k}^{r r} & \tilde{\Gamma}_{k}^{r s} & \tilde{T}_{k}^{r r} & \tilde{T}_{k}^{r s} \\
\Gamma_{k}^{s r} & \tilde{\Gamma}_{k}^{s s} & T_{k}^{s r} & \tilde{T}_{k}^{s s}
\end{array}\right] \mathrm{d} \omega(t)=Q_{k}^{-1} P_{k} \mathrm{~d} \omega(t) Q_{k}+\mathrm{d}\left[Q_{k}^{-1}\right] .
$$

Remark 2.3. The Step I basis refinement is essentially an algebraic column Gaussian elimination on the sub-matrices $\Lambda_{k}:=\left[\begin{array}{cc}\Lambda_{k}^{r r} & \Lambda_{k}^{r s} \\ \Lambda_{k}^{s r} & \Lambda_{k}^{s s}\end{array}\right]$ and $V_{k}:=\left[\begin{array}{cc}V_{k}^{r r} & V_{k}^{r s} \\ V_{k}^{s r} & V_{k}^{s s}\end{array}\right]$ to eliminate the off-diagonal blocks $\left[\Lambda_{k}^{r s}\right]$ and $\left[V_{k}^{r s}\right]$. 


\subsection{Basis refinement Step II}

This step depresses the magnitudes of $\Lambda_{k}^{s r}$ and $\Gamma_{k}^{s r}$ in $\tilde{P}_{k}$ of (18), and thus weakens the coupling of slow mode amplitudes from the fast. To this end, let

$$
\tilde{Q}_{k}=\left[\begin{array}{cccc}
I_{m} & 0 & 0 & 0 \\
-\Lambda_{k}^{s r}\left[\tilde{\Lambda}_{k}^{r r}\right]^{-1} & I_{N-m} & 0 & 0 \\
0 & 0 & I_{m} & 0 \\
0 & 0 & -\Gamma_{k}^{s r}\left[\tilde{\Gamma}_{k}^{r r}\right]^{-1} & I_{N-m}
\end{array}\right],
$$

and update $\tilde{\ell}_{k}$ to $\ell_{k+1}=\tilde{Q}_{k} \tilde{\ell}_{k}$, i.e., $\ell_{k+1}=\left(\left[\boldsymbol{f}_{(k+1)}^{r}\right]^{T},\left[\boldsymbol{f}_{(k+1)}^{s}\right]^{T},\left[\boldsymbol{g}_{(k+1)}^{r}\right]^{T},\left[\boldsymbol{g}_{(k+1)}^{s}\right]^{T}\right)$, where

$$
\boldsymbol{f}_{(k+1)}^{s}=\boldsymbol{f}_{(k)}^{s}-\Lambda_{k}^{s r}\left[\tilde{\Lambda}_{k}^{r r}\right]^{-1} \boldsymbol{f}_{(k+1)}^{r}, \quad \boldsymbol{g}_{(k+1)}^{s}=\boldsymbol{g}_{(k)}^{s}-\Gamma_{k}^{s r}\left[\tilde{\Gamma}_{k}^{r r}\right]^{-1} \boldsymbol{g}_{(k+1)}^{r} .
$$

Accordingly the basis vectors are updated by

$$
\begin{array}{rlrl}
B_{k+1}^{s} & =B_{k}^{s}-\Lambda_{k}^{s r}\left[\tilde{\Lambda}_{k}^{r r}\right]^{-1} B_{k+1}^{r}, & & \mathcal{B}_{k+1}^{s}=\mathcal{B}_{k}^{s}-\Gamma_{k}^{s r}\left[\tilde{\Gamma}_{k}^{r r}\right]^{-1} \mathcal{B}_{k+1}^{r}, \\
A_{k+1}^{r}=\left[I_{N}-A_{k+1}^{s} B_{k+1}^{s}\right] A_{k}^{r}, & & \mathcal{A}_{k+1}^{r}=\left[I_{N}-\mathcal{A}_{k+1}^{s} \mathcal{B}_{k+1}^{s}\right] \mathcal{A}_{k}^{r} .
\end{array}
$$

Following similar calculations on evolutions of $\tilde{\ell}_{k}$, we obtain the evolution of $\ell_{k+1}$ as

$$
\mathrm{d} \ell_{k+1}=P_{k+1} \mathrm{~d} \omega(t) \ell_{k+1},
$$

and

$$
P_{k+1} \mathrm{~d} \omega(t)=\tilde{Q}_{k} \tilde{P}_{k} \mathrm{~d} \omega(t) \tilde{Q}_{k}^{-1}+\mathrm{d}\left[\tilde{Q}_{k}\right]=\left[\begin{array}{cccc}
\Lambda_{k+1}^{r r} & \Lambda_{k+1}^{r s} & V_{k+1}^{r r} & V_{k+1}^{r s} \\
\Lambda_{k+1}^{s r} & \Lambda_{k+1}^{s s} & V_{k+1}^{s r} & V_{k+1}^{s s} \\
\Gamma_{k+1}^{r r} & \Gamma_{k+1}^{r s} & T_{k+1}^{r r} & T_{k+1}^{r s} \\
\Gamma_{k+1}^{s r} & \Gamma_{k+1}^{s s} & T_{k+1}^{s r} & T_{k+1}^{s s}
\end{array}\right] \mathrm{d} \omega(t),
$$

where

$$
\begin{aligned}
\Lambda_{k+1}^{r r}= & \tilde{\Lambda}_{k}^{r r}+\tilde{\Lambda}_{k}^{r s} \Lambda_{k}^{s r}\left[\tilde{\Lambda}_{k}^{r r}\right]^{-1}, & & V_{k+1}^{r r}=\tilde{V}_{k}^{r r}+\tilde{V}_{k}^{r s} \Gamma_{k}^{s r}\left[\tilde{\Gamma}_{k}^{r r}\right]^{-1} \\
\Lambda_{k+1}^{r s}= & \tilde{\Lambda}_{k}^{r s}, & V_{k+1}^{r s}= & \tilde{V}_{k}^{r s} \\
\Lambda_{k+1}^{s r}= & \Lambda_{k+1}^{s s} \Lambda_{k}^{s r}\left[\tilde{\Lambda}_{k}^{r r}\right]^{-1} & V_{k+1}^{s r}= & V_{k}^{s r}-\Lambda_{k}^{s r}\left[\tilde{\Lambda}_{k}^{r r}\right]^{-1} \tilde{V}_{k}^{r r} \\
& -\frac{\mathrm{d}}{\mathrm{d} t}\left(\Lambda_{k}^{s r}\left[\tilde{\Lambda}_{k}^{r r}\right]^{-1}\right), & & +V_{k+1}^{s s} \Gamma_{k}^{s r}\left[\tilde{\Gamma}_{k}^{r r}\right]^{-1}, \\
\Lambda_{k+1}^{s s}= & \tilde{\Lambda}_{k}^{s s}-\Lambda_{k}^{s r}\left[\tilde{\Lambda}_{k}^{r r}\right]^{-1} \tilde{\Lambda}_{k}^{r s}, & V_{k+1}^{s s}= & \tilde{V}_{k}^{s s}-\Lambda_{k}^{s r}\left[\tilde{\Lambda}_{k}^{r r}\right]^{-1} \tilde{V}_{k}^{r s},
\end{aligned}
$$




$$
\begin{array}{rlrl}
\Gamma_{k+1}^{r r}=\tilde{\Gamma}_{k}^{r r}+\tilde{\Gamma}_{k}^{r s} \Lambda_{k}^{s r}\left[\tilde{\Lambda}_{k}^{r r}\right]^{-1}, & T_{k+1}^{r r} & =\tilde{T}_{k}^{r r}+\tilde{T}_{k}^{r s} \Gamma_{k}^{s r}\left[\tilde{\Gamma}_{k}^{r r}\right]^{-1}, \\
\Gamma_{k+1}^{r s}=\tilde{\Gamma}_{k}^{r s}, & T_{k+1}^{r s}= & \tilde{T}_{k}^{r s} \\
\Gamma_{k+1}^{s r}=\Gamma_{k+1}^{s s} \Lambda_{k}^{s r}\left[\tilde{\Lambda}_{k}^{r r}\right]^{-1}, & T_{k+1}^{s r}= & T_{k}^{s r}-\frac{\mathrm{d}}{\mathrm{d} w(t)}\left(\Gamma_{k}^{s r}\left[\tilde{\Gamma}_{k}^{s r}\right]^{-1}\right) \\
& -\Gamma_{k}^{s r}\left[\Gamma_{k}^{r r}\right]^{-1} \tilde{T}_{k}^{r r}+T_{k+1}^{s s} \Gamma_{k}^{s r}\left[\tilde{\Gamma}_{k}^{r r}\right]^{-1}, \\
\Gamma_{k+1}^{s s}=\tilde{\Gamma}_{k}^{s s}-\Gamma_{k}^{s r}\left[\tilde{\Gamma}_{k}^{r r}\right]^{-1} \tilde{\Gamma}_{k}^{r s}, & T_{k+1}^{s s}=\tilde{T}_{k}^{s s}-\Gamma_{k}^{s r}\left[\tilde{\Gamma}_{k}^{r r}\right]^{-1} \tilde{T}_{k}^{r s} .
\end{array}
$$

Remark 2.4. The Step II basis refinement is essentially an algebraic row Gaussian elimination on the sub-matrices $\Lambda_{k}:=\left[\begin{array}{cc}\Lambda_{k}^{r r} & \Lambda_{k}^{r s} \\ \Lambda_{k}^{s r} & \Lambda_{k}^{s s}\end{array}\right]$ and $\Gamma_{k}:=\left[\begin{array}{cc}\Gamma_{k}^{r r} & \Gamma_{k}^{r s} \\ \Gamma_{k}^{s r} & \Gamma_{k}^{s s}\end{array}\right]$ to eliminate the off-diagonal blocks $\left[\Lambda_{k}^{s r}\right]$ and $\left[\Gamma_{k}^{s r}\right]$.

\subsection{Stochastic dynamics of the simplified system}

After $k$ iterations of refinements of SCSP, the "drift" $\boldsymbol{f}$ and the "volatility" $\boldsymbol{g}$ will be split into fast components $\boldsymbol{f}_{(k)}^{r}, \boldsymbol{g}_{(k)}^{r}$ and slow components $\boldsymbol{f}_{(k)}^{s}, \boldsymbol{g}_{(k)}^{s}$, respectively. Accordingly, the $\boldsymbol{x}$-domain is resolved into deterministic fast and slow subdomain spanned by $A_{k}^{r}$ and $A_{k}^{s}$ respectively, and stochastic fast and slow subdomain spanned by $\mathcal{A}_{k}^{r}$ and $\mathcal{A}_{k}^{s}$ respectively. Here we define formally the $k$-th stochastic slow manifold by a stochastic relation that captures the near-equilibration of fast processes. Let us define, $\forall t \geq 0$,

$$
\begin{aligned}
& \delta_{k}^{r} \boldsymbol{z}(t)=\int_{0}^{t} A_{k}^{r} \boldsymbol{f}_{(k)}^{r} \mathrm{~d} s+\mathcal{A}_{k}^{r} \boldsymbol{g}_{(k)}^{r} \circ \mathrm{d} W(s) \\
& \delta_{k}^{s} \boldsymbol{z}(t)=\int_{0}^{t} A_{k}^{s} \boldsymbol{f}_{(k)}^{s} \mathrm{~d} s+\mathcal{A}_{k}^{s} \boldsymbol{g}_{(k)}^{s} \circ \mathrm{d} W(s)
\end{aligned}
$$

such that

$$
\boldsymbol{z}(t)=\boldsymbol{z}_{0}+\delta_{k}^{s} \boldsymbol{z}(t)+\delta_{k}^{r} \boldsymbol{z}(t)
$$

We thus define the $k$-th stochastic slow manifold with

$$
\left|\delta_{k}^{r} \boldsymbol{z}(t)\right| \ll\left|\delta_{k}^{s} \boldsymbol{z}(t)\right|
$$

Note that this definition is only valid while the stochastic integrals (23) and (24) exist. Nevertheless it holds if and only if for any $t \geq 0$ and any initial condition $\boldsymbol{z}_{0}=\boldsymbol{z}(0)$,

$$
\boldsymbol{z}(t) \approx \boldsymbol{z}_{0}+\delta_{k}^{s} \boldsymbol{z}(t)
$$

i.e. the reduced dynamics of the original SDE (2) can be approximated by the desired $k$-th simplified system

$$
\mathrm{d} \boldsymbol{z} \approx A_{k}^{s} \boldsymbol{f}_{(k)}^{s} \mathrm{~d} t+\mathcal{A}_{k}^{s} \boldsymbol{g}_{(k)}^{s} \circ \mathrm{d} W(t)
$$

while approaching the " $k$-th stochastic slow manifold". 


\subsection{Explicit time integration of stiff SDE systems by SCSP}

In this subsection we build a new time-scale splitting, explicit algorithm to integrate stiff SDEs that consists of three steps. Here the time domain of interest is chosen according to the determinisitc dynamics, i.e., dynamics governed by the drift term $f$ of the stiff system (2). To this end, suppose the Jacobian matrix of $\boldsymbol{f}$ has $N$ eigenvalues with negative real parts and located away from the imaginary axis, ordered in a descending magnitude:

$$
\left|\lambda_{1}\right|>\cdots>\left|\lambda_{m}\right| \gg\left|\lambda_{m+1}\right|>\cdots\left|\lambda_{N}\right|,
$$

with the magnitude of the first $m$ eigenvalues much larger than the remaining $N-m$. Then the time domain of interest is of order $1 / \lambda_{m+1}:=\tau_{m+1}$ where stiffness of system (2) appears. The explicit algorithm proceeds as follows.

(i) Identify the number $m$ of exhausted modes at a given time. This is done by assuming that the source term $\boldsymbol{f}$ dominates the stochastic source term $\boldsymbol{g}$, and applying a similar criterion used in [73] for CSP to the deterministic part of (2). More precisely, define an error vector $\overrightarrow{\mathscr{E} z}:=\left(\mathscr{E}_{1}^{z}, \ldots, \mathscr{E}_{N}^{\boldsymbol{z}}\right)$ where $\mathscr{E}_{i}^{\boldsymbol{z}}=\varepsilon_{i}^{\text {rel }}\left|\boldsymbol{z}_{i}\right|+\varepsilon_{i}^{\text {abs }}$ for $i=1, \cdots, N$ with $\varepsilon_{i}^{\text {rel }}$ and $\varepsilon_{i}^{\text {abs }}$ representing the maximum relative and absolute errors on the $i$-th variable, respectively. The number $m$ is defined as the largest integer lying between 1 and $N$ that satisfies

$$
\left|\int_{0}^{\tau_{m+1}} \mathcal{A}^{r} \boldsymbol{g}^{r} \circ \mathrm{d} W(s)\right| \leq\left|\tau_{m+1} A^{r} \boldsymbol{f}^{r}\right|<\overrightarrow{\mathscr{E} \boldsymbol{z}} \quad \text { a.s. }
$$

(ii) Starting from a trial set of basis vectors, construct SCSP basis vectors iteratively by the procedures developed in Subsections 2.1 and 2.2.

(iii) After $k$ iterations of refinements $(k \geq 0)$, integrate the system (2) from $t_{j}$ to $t_{j+1}$ in two steps according to

$$
\begin{aligned}
\hat{\boldsymbol{z}}_{j} & =\boldsymbol{z}_{j}+\int_{t_{j}}^{t_{j+1}} A_{k}^{r} \boldsymbol{f}_{(k)}^{r} \mathrm{~d} t+\mathcal{A}_{k}^{r} \boldsymbol{g}_{(k)}^{r} \circ \mathrm{d} W(t), \\
\boldsymbol{z}_{j+1} & =\hat{\boldsymbol{z}}_{j}+\int_{t_{j}}^{t_{j+1}} A_{k}^{s} \boldsymbol{f}_{(k)}^{s} \mathrm{~d} t+\mathcal{A}_{k}^{s} \boldsymbol{g}_{(k)}^{s} \circ \mathrm{d} W(t) .
\end{aligned}
$$

Step 1. Approximating (30) by a stochastic algebraic equation. To this end, let $\tau$ be the expected elapsed time of the fast modes, i.e., the time needed for the solution trajectories of the original system to enter the neighborhood of the $k$-th stochastic slow manifold. We expect that the fast change in the solution over the time interval $\left[t_{j}, t_{j+1}\right]$ is dominated by that change occuring in the interval $\left[t_{j}, t_{j}+\tau\right]$ when the fast processes are decaying quickly. In fact, due to (26)

$$
\left|\int_{t_{j}+\tau}^{t_{j+1}} A_{k}^{r} \boldsymbol{f}_{(k)}^{r} \mathrm{~d} t+\mathcal{A}_{k}^{r} \boldsymbol{g}_{(k)}^{r} \circ \mathrm{d} W(t)\right| \ll\left|\int_{t_{j}+\tau}^{t_{j+1}} A_{k}^{s} \boldsymbol{f}_{(k)}^{s} \mathrm{~d} t+\mathcal{A}_{k}^{s} \boldsymbol{g}_{(k)}^{s} \circ \mathrm{d} W(t)\right| .
$$


Hence (30) can be approximated by

$$
\hat{\boldsymbol{z}}_{j} \approx \boldsymbol{z}_{j}+\int_{t_{j}}^{t_{j}+\tau} A_{k}^{r} \boldsymbol{f}_{(k)}^{r} \mathrm{~d} t+\mathcal{A}_{k}^{r} \boldsymbol{g}_{(k)}^{r} \circ \mathrm{d} W(t) .
$$

Using the Euler method for $\int_{t_{j}}^{t_{j}+\tau} A_{k}^{r} \boldsymbol{f}_{(k)}^{r} \mathrm{~d} t$, and the midpoint rule (see e.g., $[18,53])$ for $\int_{t_{j}}^{t_{j}+\tau} \mathcal{A}_{k}^{r} \boldsymbol{g}_{(k)}^{r} \circ \mathrm{d} W(t)(33)$ then becomes

$$
\hat{\boldsymbol{z}}_{j} \approx \boldsymbol{z}_{j}+A_{k}^{r}\left(\boldsymbol{z}_{j}\right) \boldsymbol{f}_{(k)}^{r}\left(\boldsymbol{z}_{j}\right) \tau+\frac{\xi \sqrt{\tau}}{2}\left(\mathcal{A}_{k}^{r}\left(\boldsymbol{z}_{j}\right) \boldsymbol{g}_{(k)}^{r}\left(\boldsymbol{z}_{j}\right)+\mathcal{A}_{k}^{r}\left(\hat{\boldsymbol{z}}_{j}\right) \boldsymbol{g}_{(k)}^{r}\left(\hat{\boldsymbol{z}}_{j}\right)\right),
$$

where $\xi \sim \mathcal{N}(0,1)$.

Step 2. Integrating along the $k$-th stochastic slow manifold according to the simplified system (28), to obtain the next state $\boldsymbol{z}_{j+1}$

$$
\boldsymbol{z}_{j+1} \approx \hat{\boldsymbol{z}}_{j}+\int_{t_{j}}^{t_{j+1}} A_{k}^{s} \boldsymbol{f}_{(k)}^{s} \mathrm{~d} t+\mathcal{A}_{k}^{s} \boldsymbol{g}_{(k)}^{s} \circ \mathrm{d} W(t)
$$

Similar to step 1, using the Euler method for $\int_{t_{j}}^{t_{j+1}} A_{k}^{s} \boldsymbol{f}_{(k)}^{s} \mathrm{~d} t$, and the midpoint rule for $\int_{t_{j}}^{t_{j+1}} \mathcal{A}_{k}^{s} \boldsymbol{g}_{(k)}^{s} \circ \mathrm{d} W(t)$ in (35) gives

$$
\begin{aligned}
\boldsymbol{z}_{j+1} \approx & \hat{\boldsymbol{z}}_{j}+A_{k}^{s}\left(\boldsymbol{z}_{j}\right) \boldsymbol{f}_{(k)}^{s}\left(\boldsymbol{z}_{j}\right) h_{j} \\
& +\frac{\xi \sqrt{h_{j}}}{2}\left(\mathcal{A}_{k}^{s}\left(\boldsymbol{z}_{j}\right) \boldsymbol{g}_{(k)}^{s}\left(\boldsymbol{z}_{j}\right)+\mathcal{A}_{k}^{s}\left(z_{j+1}\right) \boldsymbol{g}_{(k)}^{s}\left(z_{j+1}\right)\right),
\end{aligned}
$$

where $\xi \sim \mathcal{N}(0,1)$ and $h_{j}:=z_{j+1}-z_{j}$.

Note that usually (34) and (36) are systems of implicit nonlinear algebraic equations, and can be solved by iteration methods, etc.

Remark 2.5. For CSP with $\boldsymbol{g}^{s}=\boldsymbol{g}^{r} \equiv 0, \tau$ is of the scale $1 /\left|\lambda_{m}\right|$ where $\lambda_{m}$ is the smallest-in-magnitude eigenvalue of the fast sub-system. Thanks to [4, 5] we could assume that $\tau$ is still of the scale $1 /\left|\lambda_{m}\right|$ for SCSP, provided that the deterministic counterpart of the original system (2) admits a uniformly hyperbolic and asymptotically stable slow manifold.

Remark 2.6. Note that for approximation (33) to hold, the integration step size $t_{j+1}-$ $t_{j}$ needs to be no less than $\tau$. Whereas for general explicit integrators for stiff systems the step size can be at most $1 /\left|\lambda_{1}\right|$, where $\lambda_{1}$ is the largest-in-magnitude eigenvalue of the fast sub-system. More precisely general explicit integrators require an upper bound on the step size, while explicit integration by SCSP requires a lower bound. 


\section{Application of SCSP to stochastic Davis-Skodje}

In this section we will apply the proposed SCSP algorithm to the following stochastic Davis-Skodje system:

$$
\begin{aligned}
\mathrm{d} x(t) & =-x(t) \mathrm{d} t+\mu x(t) \circ \mathrm{d} W(t), \\
\mathrm{d} y(t) & =\left(-\gamma y(t)+\gamma \frac{x(t)}{1+x(t)}-\frac{x(t)}{(1+x(t))^{2}}\right) \mathrm{d} t+\sigma \sqrt{\gamma} y(t) \circ \mathrm{d} W(t),
\end{aligned}
$$

where $x(t)$ and $y(t)$ are stochastic processes, $\mu$ and $\sigma$ are small positive numbers, and $W(t)$ is a one-dimensional Wiener process. Note that the Davis-Skodje model has been used for the purpose of testing numerical model reduction approaches, but does not arise from a set of real chemical reactions. Hence the above CLEs governing the stochastic Davis-Skodje system are obtained by perturbing both the fast and slow variables of the deterministic Davis-Skodje system $[11,66]$ by multiplicative white noise, with different noise intensities due to different time scales.

First rewrite system (37) - (38) in the vector form as

$$
\mathrm{d} \boldsymbol{z}=\boldsymbol{f}(\boldsymbol{z}) \mathrm{d} t+\boldsymbol{g}(\boldsymbol{z}) \circ \mathrm{d} W(t),
$$

where

$$
\boldsymbol{z}=\left(\begin{array}{l}
y \\
x
\end{array}\right), \quad \boldsymbol{f}(\boldsymbol{z})=\left(\begin{array}{c}
-\gamma y+\gamma \frac{x}{1+x}-\frac{x}{(1+x)^{2}} \\
-x
\end{array}\right), \quad \boldsymbol{g}(\boldsymbol{z})=\left(\begin{array}{c}
\sigma \sqrt{\gamma} y \\
\mu x
\end{array}\right) .
$$

The Jacobian of $\boldsymbol{f}$ and $\boldsymbol{g}$ are given by

$$
J_{\boldsymbol{f}}=\left[\begin{array}{cc}
-\gamma & \Delta(x) \\
0 & -1
\end{array}\right], \quad J_{\boldsymbol{g}}=\left[\begin{array}{cc}
\sigma \sqrt{\gamma} & 0 \\
0 & \mu
\end{array}\right]
$$

where

$$
\Delta(x)=\frac{\gamma}{(1+x)^{2}}+\frac{x-1}{(1+x)^{3}} .
$$

\subsection{An arbitrary initial set of basis vectors}

Suppose that the initial guess for the basis vectors is

$$
\boldsymbol{a}_{(0)}^{r}=\left(\begin{array}{c}
2 \\
-1
\end{array}\right), \quad \boldsymbol{a}_{(0)}^{s}=\left(\begin{array}{c}
-2 \\
3
\end{array}\right), \quad \boldsymbol{\alpha}_{(0)}^{r}=\left(\begin{array}{c}
-3 \\
1
\end{array}\right), \quad \boldsymbol{\alpha}_{(0)}^{s}=\left(\begin{array}{c}
-1 \\
1
\end{array}\right)
$$

with associated inverse vectors

$$
\boldsymbol{b}_{(0)}^{r}=\left(\begin{array}{ll}
\frac{3}{4} & \frac{1}{2}
\end{array}\right), \boldsymbol{b}_{(0)}^{s}=\left(\begin{array}{ll}
\frac{1}{4} & \frac{1}{2}
\end{array}\right), \boldsymbol{\beta}_{(0)}^{r}=\left(\begin{array}{ll}
-\frac{1}{2} & -\frac{1}{2}
\end{array}\right), \boldsymbol{\beta}_{(0)}^{s}=\left(\begin{array}{ll}
\frac{1}{2} & \frac{3}{2}
\end{array}\right) .
$$


The corresponding fast and slow modes are

$$
\begin{aligned}
& f_{(0)}^{r}=\frac{3}{4}\left(-\gamma y+\gamma \frac{x}{1+x}-\frac{x}{(1+x)^{2}}\right)-\frac{1}{2} x, \\
& g_{(0)}^{r}=-\frac{1}{2}(\sigma \sqrt{\gamma} y+\mu x), \\
& f_{(0)}^{s}=\frac{1}{4}\left(-\gamma y+\gamma \frac{x}{1+x}-\frac{x}{(1+x)^{2}}\right)-\frac{1}{2} x, \\
& g_{(0)}^{s}=\frac{1}{2} \sigma \sqrt{\gamma} y+\frac{3}{2} \mu x .
\end{aligned}
$$

Equations (42) and (43) imply that when the fast time scale becomes exhausted $(t \gg$ $1 / \gamma)$, the fast amplitudes $f_{(0)}^{r} \sim \mathcal{O}(1), g_{(0)}^{r} \sim \mathcal{O}(\max \{\mu, \sigma \sqrt{\gamma}\})$, and hence are not negligible. In addition, the 0 -th simplified system given by (44) and (45),

$$
\begin{aligned}
& \mathrm{d} y \approx-\frac{1}{2}\left(-\gamma y+\gamma \frac{x}{1+x}-\frac{x}{(1+x)^{2}}-2 x\right) \mathrm{d} t-\left(\frac{1}{2} \sigma \sqrt{\gamma} y+\frac{3}{2} \mu x\right) \circ \mathrm{d} W(t), \\
& \mathrm{d} x \approx \frac{3}{4}\left(-\gamma y+\gamma \frac{x}{1+x}-\frac{x}{(1+x)^{2}}-2 x\right) \mathrm{d} t+\left(\frac{1}{2} \sigma \sqrt{\gamma} y+\frac{3}{2} \mu x\right) \circ \mathrm{d} W(t),
\end{aligned}
$$

is clearly stiff.

Performing one iteration of refinements as proposed in Subsections 2.1 gives the first-refined fast basis vectors

$\boldsymbol{b}_{(1)}^{r}=\left(\frac{3}{4}+\frac{1}{4} \kappa_{1}(x), \quad \frac{1}{2}+\frac{1}{2} \kappa_{1}(x)\right), \quad \boldsymbol{\beta}_{(1)}^{r}=\left(-\frac{1}{2}+\frac{1}{2} \kappa_{2}(x), \quad-\frac{1}{2}+\frac{3}{2} \kappa_{2}(x)\right)$,

where

$$
\kappa_{1}(x)=\frac{6 \gamma+9 \Delta(x)-6}{-6 \gamma-3 \Delta(x)+2}, \quad \kappa_{2}(x)=\frac{6 \gamma+6 \Delta-4}{9 \gamma+3 \Delta(x)-2} .
$$

As a result the stochastic and deterministic fast modes are

$$
\begin{aligned}
& f_{(1)}^{r}=\left(\frac{3}{4}+\frac{1}{4} \kappa_{1}(x)\right)\left(-\gamma y+\gamma \frac{x}{1+x}-\frac{x}{(1+x)^{2}}\right)-\frac{1}{2} x\left(1+\kappa_{1}(x)\right),( \\
& g_{(1)}^{r}=\left(-\frac{1}{2}+\frac{1}{2} \kappa_{2}(x)\right) \sigma \sqrt{\gamma} y+\left(-\frac{1}{2}+\frac{3}{2} \kappa_{2}(x)\right) \mu x
\end{aligned}
$$

Simplifying $f_{(1)}^{r}$ implies that when the fast time scale becomes exhausted the deterministic fast amplitude $f_{(1)}^{r} \sim \mathcal{O}(1 / \gamma)$. The stochastic fast amplitude $g_{(1)}^{r}$ still has an order of $\mathcal{O}(\max \{\mu, \sigma \sqrt{\gamma}\})$, but is smaller than $g_{(0)}^{r}$ (see detailed calculations in Appendix).

On the other hand, the first-refined slow basis vectors are

$$
\boldsymbol{a}_{(1)}^{s}=\left(\begin{array}{c}
-2-2 \kappa_{1}(x) \\
\frac{1}{2} \kappa_{1}(x)+3
\end{array}\right), \quad \boldsymbol{\alpha}_{(1)}^{s}=\left(\begin{array}{c}
-1+\frac{3}{2} \kappa_{2}(x) \\
1-\frac{1}{2} \kappa_{2}(x)
\end{array}\right) .
$$


Though complicated, $f_{(1)}^{s}$ and $g_{(1)}^{s}$ can still be calculated analytically and the magnitudes of $\boldsymbol{a}_{(1)}^{s} f_{(1)}^{s}$ and $\boldsymbol{\alpha}_{(1)}^{s} g_{(1)}^{s}$ are both of order 1. This implies that the first simplified system is no longer stiff.

In summary, starting from the arbitrary initial set of basis vectors, the effects of first iteration of refinements include:

1. reducing the order of magnitude of $f^{r}$ by $1 / \gamma$;

2. reducing the magnitude of $g^{r}$;

3. reducing the stiffness of the simplified system.

\subsection{Eigenvectors as initial set of basis vectors}

At any point $(y, x)$, the eigenvalues of $J_{\boldsymbol{f}}$ are $-\gamma$ and -1 , and the corresponding eigenvectors are $(1,0)^{T}$ and $(\Delta(x), \gamma-1)^{T}$. Hence we pick the trial basis for $\boldsymbol{f}$ to be

$$
\boldsymbol{a}_{(0)}^{r}:=\left(\begin{array}{c}
1 \\
0
\end{array}\right), \quad \boldsymbol{a}_{(0)}^{s}:=\left(\begin{array}{c}
\Delta(x) \\
\gamma-1
\end{array}\right),
$$

with associated inverse vectors

$$
\boldsymbol{b}_{(0)}^{r}=\left(\begin{array}{ll}
1 & -\frac{\Delta(x)}{\gamma-1}
\end{array}\right), \quad \boldsymbol{b}_{(0)}^{s}=\left(\begin{array}{ll}
0 & \frac{1}{\gamma-1}
\end{array}\right) .
$$

Similarly the eigenvalues of $J_{\boldsymbol{g}}$ are $\sigma \sqrt{\gamma}$ and $\mu$ and the corresponding eigenvectors (trial basis for $\boldsymbol{g}$ ) and their inverses are

$$
\boldsymbol{\alpha}_{(0)}^{r}=\left(\begin{array}{c}
1 \\
0
\end{array}\right), \quad \boldsymbol{\alpha}_{(0)}^{s}=\left(\begin{array}{l}
0 \\
1
\end{array}\right), \quad \boldsymbol{\beta}_{(0)}^{r}=\left(\begin{array}{ll}
1 & 0
\end{array}\right), \quad \boldsymbol{\beta}_{(0)}^{s}=\left(\begin{array}{ll}
0 & 1
\end{array}\right) .
$$

Therefore the deterministic and stochastic fast and slow modes can be calculated to be

$$
\begin{aligned}
& f_{(0)}^{r}=\boldsymbol{b}_{(0)}^{r} \cdot \boldsymbol{f}=-\gamma y+\gamma \frac{x}{1+x}-\frac{x}{(1+x)^{2}}+\frac{\Delta(x)}{\gamma-1} x, \\
& f_{(0)}^{s}=\boldsymbol{b}_{(0)}^{s} \cdot \boldsymbol{f}=-\frac{1}{\gamma-1} x, \\
& g_{(0)}^{r}=\boldsymbol{\beta}_{(0)}^{r} \cdot \boldsymbol{g}=\sigma \sqrt{\gamma} y, \\
& g_{(0)}^{s}=\boldsymbol{\beta}_{(0)}^{s} \cdot \boldsymbol{g}=\mu x .
\end{aligned}
$$

Equations (52) and (54) imply that when the fast time scale becomes exhausted, the deterministic fast amplitude $f_{(0)}^{r} \sim \mathcal{O}(1 / \gamma)$ and the stochastic fast amplitude $g_{(0)}^{r} \sim$ $\mathcal{O}(\sigma \sqrt{\gamma})$. In addition, the 0-th simplified system calculated from (49), (50), (53) and (55) reads

$$
\begin{aligned}
\mathrm{d} y & \approx-\frac{1}{\gamma-1} \Delta(x) x \mathrm{~d} t \\
\mathrm{~d} x & \approx-x \mathrm{~d} t+\mu x \circ \mathrm{d} W(t),
\end{aligned}
$$


which is already non-stiff, and, in fact, satisfies the relation (26). Thus the second step of refinement (to reduce the stiffness) is not needed. In fact, due to the special structure of this system (see detailed calculations in Appendix),

$$
\begin{array}{llll}
\boldsymbol{b}_{(k)}^{r} \equiv \boldsymbol{b}_{(0)}^{r}, & \boldsymbol{b}_{(k)}^{s} \equiv \boldsymbol{b}_{(0)}^{s}, & \boldsymbol{\beta}_{(k)}^{s} \equiv \boldsymbol{\beta}_{(0)}^{s}, & \forall k \geq 0, \\
\boldsymbol{a}_{(k)}^{r} \equiv \boldsymbol{a}_{(0)}^{r}, & \boldsymbol{a}_{(k)}^{s} \equiv \boldsymbol{a}_{(0)}^{s}, & \boldsymbol{\alpha}_{(k)}^{r} \equiv \boldsymbol{\alpha}_{(0)}^{r}, & \forall k \geq 0,
\end{array}
$$

and as a result

$$
f_{(k)}^{r} \equiv f_{(0)}^{r}, \quad f_{(k)}^{s} \equiv f_{(0)}^{s}, \quad g_{(k)}^{s} \equiv g_{(0)}^{s}, \quad \forall k \geq 1 .
$$

After one iteration of refinement,

$$
\boldsymbol{\beta}_{(1)}^{r}=\left(\begin{array}{ll}
1 & -\frac{\Delta(x)}{\gamma-1}
\end{array}\right), \quad \boldsymbol{\alpha}_{(1)}^{s}=\left(\begin{array}{c}
\frac{\Delta(x)}{\gamma-1} \\
1
\end{array}\right)
$$

and

$$
g_{(1)}^{r}=\sigma \sqrt{\gamma} y-\frac{\mu}{\gamma-1} x \Delta(x) .
$$

Therefore if $\mathcal{O}(\mu) \leq \mathcal{O}(\sigma \sqrt{\gamma})$, the magnitude of $g_{(1)}^{r}$ is less than the magnitude of $g_{(1)}^{r}$. On the other hand the first simplified system becomes

$$
\begin{aligned}
& \mathrm{d} y \approx-\frac{1}{\gamma-1} \Delta(x) x \mathrm{~d} t+\frac{\Delta(x)}{\gamma-1} \circ \mathrm{d} W(t), \\
& \mathrm{d} x \approx-x \mathrm{~d} t+\mu x \circ \mathrm{d} W(t) .
\end{aligned}
$$

In summary, due to (56) and (57) the eigenvectors are close to optimal vectors as the initial set of basis. As a result, the first refinement does not further reduce the magnitude of $f^{r}$ or the stiffness of the simplified system. However, it still reduces the magnitude of $g^{r}$, provided $\mathcal{O}(\mu) \leq \mathcal{O}(\sigma \sqrt{\gamma})$.

\subsection{Explicit time integration using eigenvectors}

Assume the system starts from initial value $\boldsymbol{z}_{0}=\left(y_{0}, x_{0}\right)$ at time $t_{0}$ and at time $t_{j}$ the system is at the point $\boldsymbol{z}_{j}=\left(y_{j}, x_{j}\right)$. We will integrate the system forward from $t_{j}$ to $t_{j+1}$ and obtain $\boldsymbol{z}_{j+1}=\left(y_{j+1}, x_{j+1}\right)$ in two steps.

For the stochastic Davis-Skodje model, it is clear that $m=1$. In fact, $\left|\lambda_{2}\right|=1$ and the condition (29) is satisfied with $\tau_{2}=1$, based on numerical experiments. Hence $\left|\lambda_{m}\right|=\left|\lambda_{1}\right|=\gamma$ and we pick $\tau=\frac{1}{\gamma}$ and assume $h_{j} \equiv h \geq \tau$. Then by (34)

$$
\hat{\boldsymbol{z}}_{j} \approx \boldsymbol{z}_{j}+\frac{1}{\gamma} \boldsymbol{a}_{(0)}^{r} f_{(0)}^{r}\left(\boldsymbol{z}_{j}\right)+\frac{\xi}{2 \sqrt{\gamma}} \boldsymbol{\alpha}_{(0)}^{r}\left(g_{(0)}^{r}\left(\boldsymbol{z}_{j}\right)+g_{(0)}^{r}\left(\hat{\boldsymbol{z}}_{j}\right)\right),
$$

where $\xi \sim \mathcal{N}(0,1)$. The approximation (60) is valid, as the assumption (32) is found to be satisfied, numerically. Based on (49), (51), (52) and (54), we have

$$
\begin{aligned}
& \hat{x}_{j} \approx x_{j} \\
& \hat{y}_{j} \approx \frac{2}{2-\sigma \xi}\left(\frac{\sigma \xi}{2} y_{j}+\frac{x_{j}}{1+x_{j}}-\frac{x_{j}}{\gamma\left(1+x_{j}\right)^{2}}+\frac{\Delta_{j} x_{j}}{\gamma(\gamma-1)}\right),
\end{aligned}
$$


where $\Delta_{j}=\Delta\left(x_{j}\right)$.

By (36) the second step reads

$$
\boldsymbol{z}_{j+1} \approx \hat{\boldsymbol{z}}_{j}+\boldsymbol{a}_{(0)}^{s} f_{(0)}^{s}\left(\boldsymbol{z}_{j}\right) h+\frac{\xi \sqrt{h}}{2} \boldsymbol{\alpha}_{(0)}^{s}\left(g_{(0)}^{s}\left(\boldsymbol{z}_{j}\right)+g_{(0)}^{s}\left(\boldsymbol{z}_{j+1}\right)\right) .
$$

With the data in (49), (51), (53) and (55), we obtain

$$
\begin{aligned}
x_{j+1} & \approx \frac{2}{2-\mu \xi \sqrt{h}}\left(\hat{x}_{j}-x_{j} h+\frac{\mu \xi \sqrt{h}}{2} x_{j}\right), \\
y_{j+1} & \approx \hat{y}_{j}-\frac{\Delta_{j}}{\gamma-1} x_{j} h .
\end{aligned}
$$

Similarly, the explicit time integration using eigenvectors after the first refinement can be calculated to be

$$
\begin{aligned}
& \hat{x}_{j} \approx x_{j}, \\
& \hat{y}_{j} \approx \frac{2}{2-\sigma \xi}\left(\frac{\sigma \xi}{2} y_{j}+\frac{x_{j}}{1+x_{j}}+\frac{2 x_{j}^{2}}{\gamma(\gamma-1)\left(1+x_{j}\right)^{3}}-\frac{\mu \xi \Delta_{j} x_{j}}{\sqrt{\gamma}(\gamma-1)}\right) .
\end{aligned}
$$

and

$$
\begin{aligned}
& x_{j+1} \approx \frac{2}{2-\mu \xi \sqrt{h}}\left(\hat{x}_{j}-x_{j} h+\frac{\mu \xi \sqrt{h}}{2} x_{j}\right), \\
& y_{j+1} \approx \hat{y}_{j}-\frac{\Delta_{j} x_{j}}{\gamma-1} h+\frac{1}{2}\left(\frac{\Delta_{j}}{\gamma-1} \mu x_{j}+\left(\frac{\Delta_{j+1}}{\gamma}+\frac{\Delta_{j}}{\gamma(\gamma-1)}\right) \mu x_{j+1}\right) \xi \sqrt{h} .
\end{aligned}
$$

where $\Delta_{j+1}=: \Delta\left(x_{j+1}\right)$.

\subsection{Numerical Illustration}

In the following, we illustrate the numerical application of SCSP to the stiff DS system. We begin by examining the response of the system as computed with conventional explicit time stepping at sufficiently small time steps. Specifically, with $\sigma=\mu=0.02$, $\gamma=100$, and $h=10^{-4}$, Figure 1 shows state trajectories starting at a number of initial conditions in state space. We show both the SDE trajectories and those from the deterministic DS ODE system. The deterministic manifold is also indicated. This plot illustrates the general character of both deterministic and stochastic fast/slow systems, where, for any initial condition that's away from the manifold, the state evolves first (with the fast processes) toward the manifold, and then subsequently evolves (with the slow processes) along the manifold, in the deterministic case, or in the near vicinity of the manifold, in the stochastic case. In particular, while, in the deterministic case, the fast time scales are "exhausted" once the solution reaches the manifold, this does not happen in the stochastic case. In this latter case, fast processes are continually excited by the randomness of the diffusion term of the SDE. However, the behavior of 
the stochastic solution, once the vicinity of the deterministic manifold is reached, is characterized by random motion within the confines of a basis of attraction around the deterministic manifold.

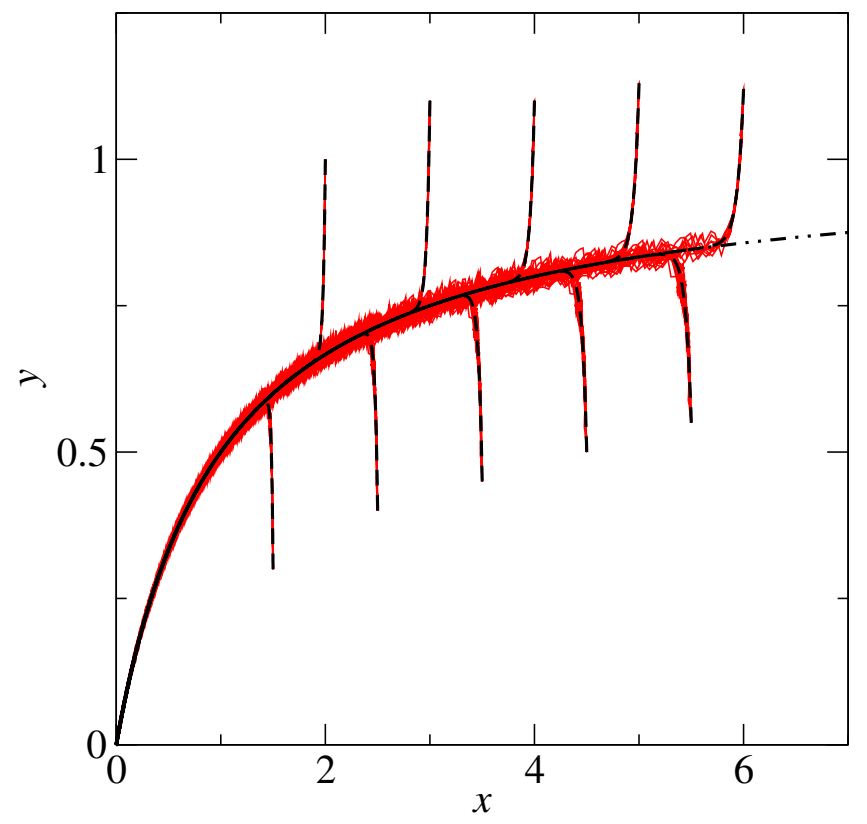

Figure 1: Solution trajectories starting from a number of initial conditions, computed with both the deterministic and stochastic DS system, using explicit time integration of the stiff system. Also shown is the deterministic system manifold. Parameter values are: $\sigma=\mu=0.02, h=10^{-4}, \gamma=100$.

Let us consider next the solution of the stochastic DS system computed both by Stratonovich time integration of the original stiff SDE system (37)-(38) (see Appendix for the scheme), and using SCSP Stratonovich time integrator relying on eigenvectors, without refinement (61)-(63). We consider again the case with $\sigma=\mu=0.02$, $\gamma=100$, and with $h_{\text {stiff }}=10^{-4}, h_{\mathrm{SCSP}}=10^{-2}$, which we refer to as the baseline case going forward. The resulting time evolution of the stiff and SCSP solutions is shown in Figure 2. The two solutions generally exhibit similar behavior for both $(x, y)$ variables. In this linear time scale, the fast initial decay of the fast variable, $y$, is not easily distinguishable. We present the same results plotted on a logarithmic time scale in Figure 3, where, now, the result of using SCSP, bypassing the small time-step integration of the fast initial transient is clearly visible in the solution, where the $h_{\mathrm{SCSP}}=0.01$ gives us the first time-step solution at $t=0.01$. Further, the utility of SCSP, which sacrifices accuracy in the computation of the fast motions, while accurately capturing the longer time scale solution dynamics, is also illustrated in this plot, as the solution is clearly incorrect for small $t$, but is generally consistent with the stiff solution for large $t$. Moreover, and as expected from earlier analysis above, we see essentially negligible difference in the solution trajectories computed with SCSP, employing the Jacobian eigenvectors as basis vectors, with or without refinement, as shown in Figure 4. 


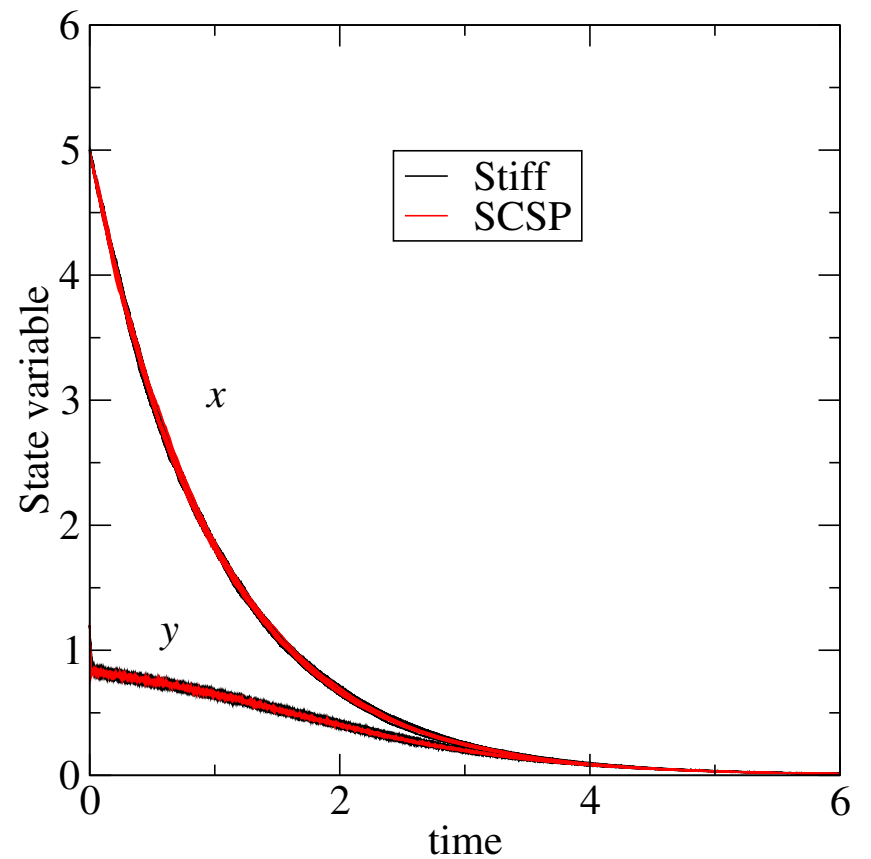

Figure 2: Time evolution of $(x, y)$, starting at $(5,1.2)$, based on computations of the stochastic DS system with both the original stiff formulation and with SCSP, with no refinement. Baseline case, with the same $(\sigma, \mu, \gamma)$ as in Fig. 1 , and with $h_{\text {stiff }}=10^{-4}$ and $h_{\mathrm{SCSP}}=10^{-2}$. 


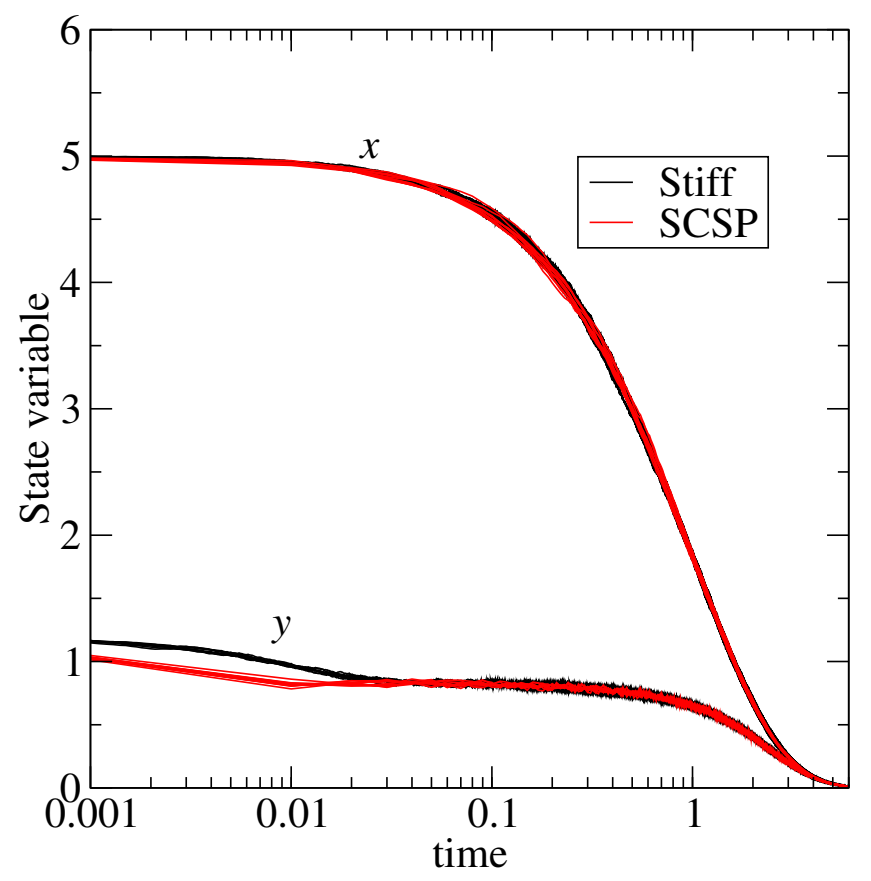

Figure 3: A plot of the same data in Fig. 2, shown on a logarithmic time scale

We also present the above time evolution of the solution, plotted in phase space, as shown in Figure 5, where we zoom-in on the early time phase. Here, we see again the result of the SCSP integrator in bringing the initial condition quickly to the vicinity of the deterministic manifold, thereby bypassing the small time-step time integration of the fast processes. We note again the negligible role of refinement, given the use of Jacobian eigenvectors as basis vectors.

In order to analyze the stochastic dynamics in more detail, we begin by examining the time evolution of the mean and standard deviation of the state variables, shown in Figures 6 and 7. The plots show results from the stiff and SCSP no-refinement cases. The results from the case with one refinement (not shown) are indistinguishable from the no-refinement case on this plot. We see that the mean behavior of both slow and fast $(x, y)$ variables is in good agreement between the two schemes. Further, the standard deviation of the slow variable $x$ is also in agreement. On the other hand, the standard deviation of the fast variable, $y$, is not well captured by SCSP. In fact, this standard deviation is significantly larger than that computed from the stiff system over a significant portion of the time trajectory. Here, we start to see the impact of the approximations made in SCSP as regards the fast processes over longer time scales. While the mean behavior of the fast variable is represented well over long time scales, being reminiscent of the slaving of the fast variables to the slow variables in stiff ODEs, there is a non-negligible error in the statistics of the fast variable, resulting from the approximations employed by SCSP in the representation of fast processes. In fact, 


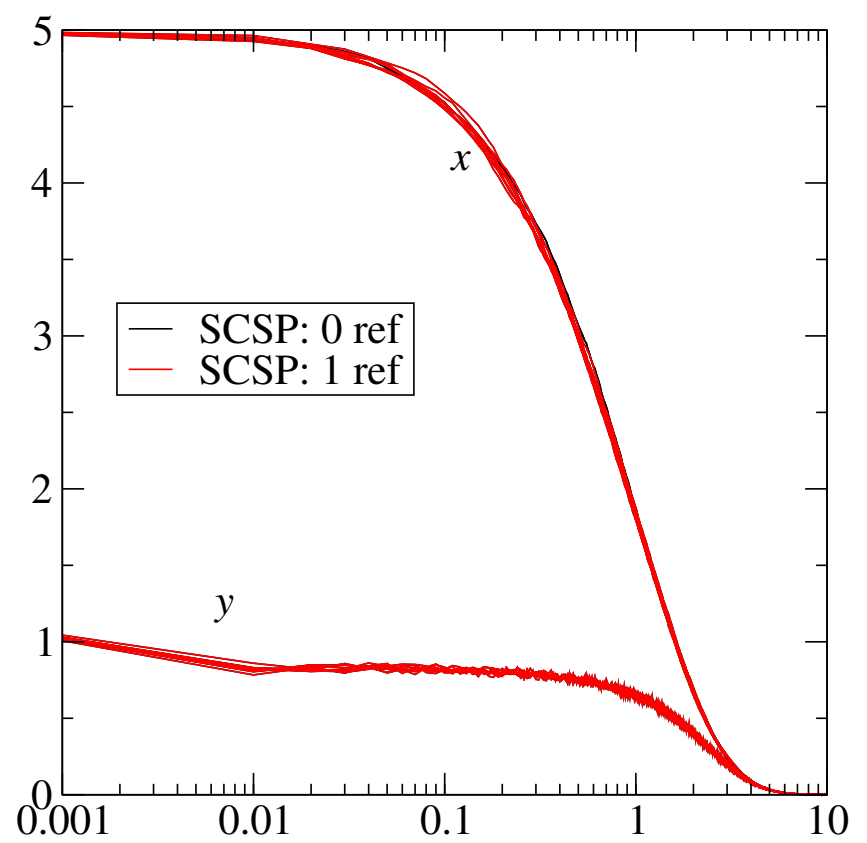

Figure 4: A comparison of the time trajectories of the stochastic DS system using the SCSP integrator including both no-refinement and one refinement cases, for the baseline conditions, and plotted on a logarithmic time axis. 


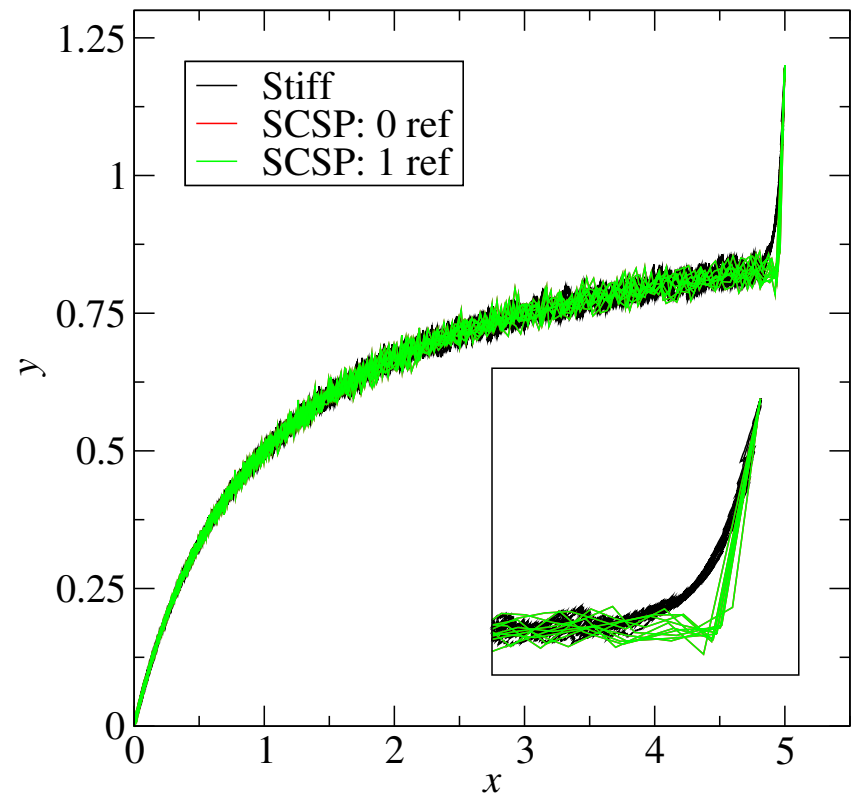

Figure 5: Evolution of $(x, y)$ in the phase plane, showing the fast evolution towards the manifold, and subsequent motion in the manifold basin, with both the stiff integration and SCSP, including cases with 0 and 1 refinements, for baseline conditions.

while reduction of the time step $h_{\mathrm{SCSP}}$ does lead to consequent reduction in the $y$-stdv, towards that of the stiff solution, we do not see asymptotic convergence of the $y$-stdv results between the two schemes, as we'll see later below. This irreducible error in $y$-stdv is due to the fact that the stochastic fast mode, $g^{r}$, even after the first refinement, is of order $\sigma \sqrt{\gamma}$, and hence is still non-negligible when the fast time scale for the drift (of order $\gamma$ ) is exhausted. However, $g^{r}$ can be neglected after a longer period of time when the fast time scale for diffusion (of order $\sqrt{\gamma}$ ) has passed, and thus the convergence of the standard deviation of $y$ by SCSP to the standard deviation by stiff SDEs. Clearly, thus, while SCSP is effective in capturing the stochastic dynamics of the slow variable, only the mean behavior of the fast variable is well captured, resulting from the approximation of the fast dynamics. This is, of course, expected, just as, in deterministic stiff ODE systems, the fast transients are not well represented with CSP.

Consider next the covariance structure of the stochastic processes $(x(t, \omega), y(t, \omega))$. We examine the eigenstructure of the covariance matrix computed from a large number of sample system trajectories $\left(10^{4}\right)$. The decay in the magnitude of the eigenvalues is shown in Figure 8, for both stiff and SCSP no-refinement schemes. Here again, the results with one refinement are on top of the no-refinement case, and are thus not shown. Unless otherwise stated, this is also the case in other results presented in the following. Considering Fig. 8, we note first that the structure of the eigenvalue spectrum of the SDE, as exemplified by the stiff solution results, indicate a distinct difference between the slow and fast variables. The slow variable exhibits spectral decay of the eigenvalue amplitude with eigenmode number, with a slower decay rate for the more energetic 


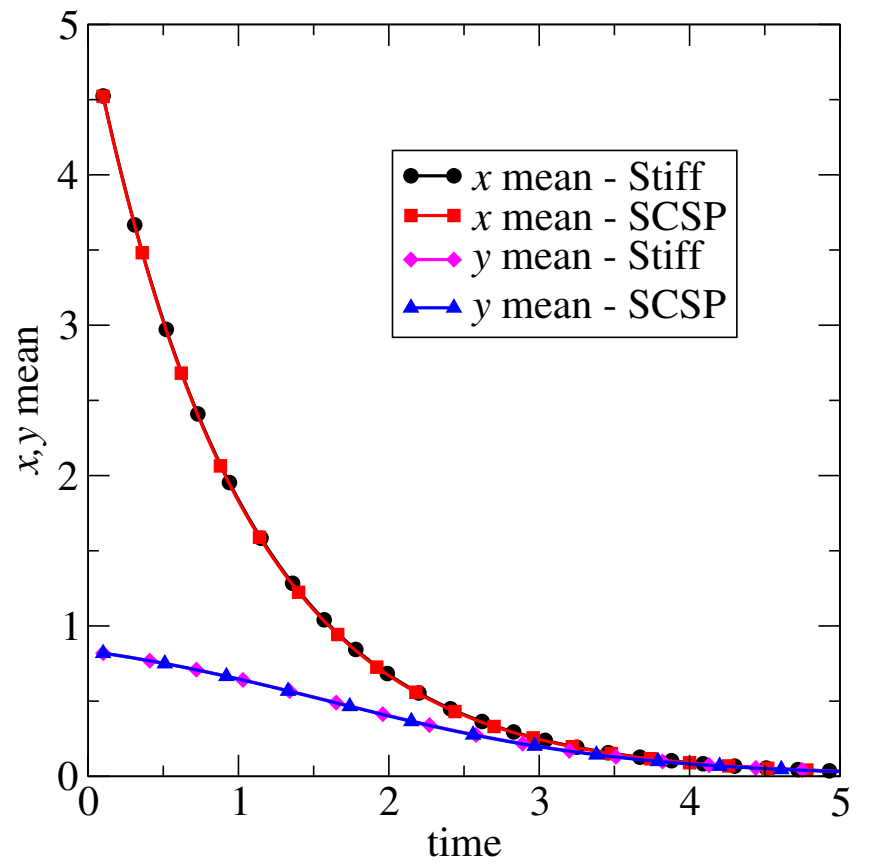

Figure 6: Time evolution of the mean of $(x, y)$ for the stiff and SCSP-no-refinement cases. Employing $10^{4}$ samples, with the baseline conditions.

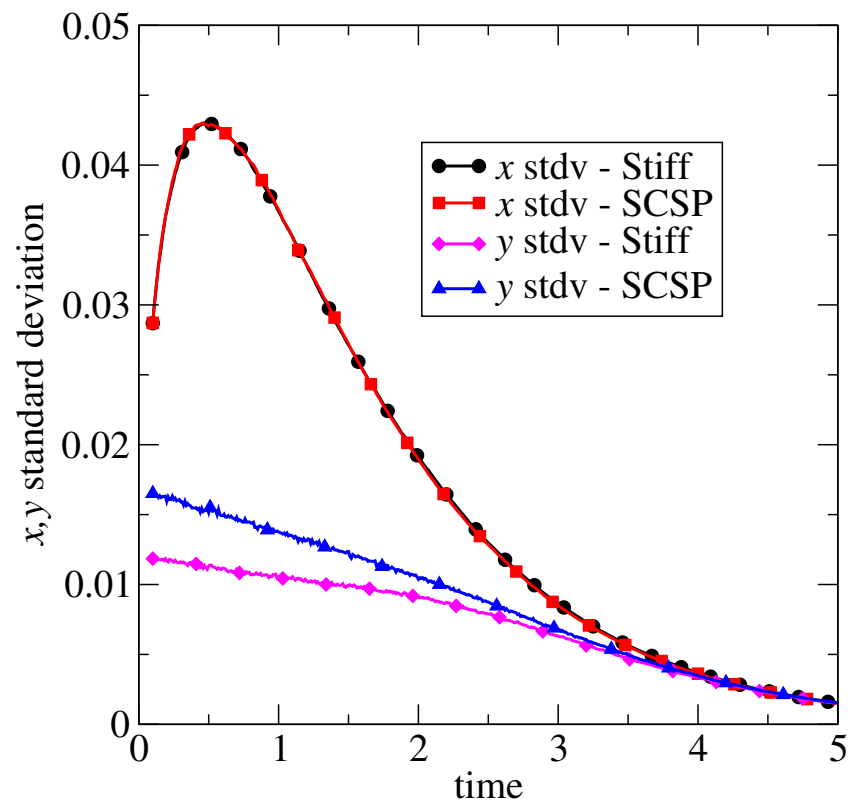

Figure 7: Time evolution of the standard deviation of $(x, y)$ for the stiff and SCSP-no-refinement cases. Employing $10^{4}$ samples, with the baseline conditions. 
eigenmodes (low eigenvalue numbers, roughly $<100$ ), and a faster deca rate for lower energy modes, beyond the 200th eigenmode. On the other hand, the eigenspectrum for the fast variable $y$ exhibits a different structure, with spectral decay rates for high and low energy modes, and a nearly flat plateau in the intermediate region. This overall picture is a function of the SDE structure, as well as its parameters $(\mu, \sigma, \gamma)$. In particular, we note that numerical experiments with order of magnitude variation in each of these parameters revealed interesting results in the spectrum (not shown). For the slow $x$ variable, we find that the spectrum is essentially insensitive to changes in $(\sigma, \gamma)$, but exhibits strong dependence on $\mu$. In particular, increasing $\mu$ from 0.02 to 0.2 increases the amplitude of the $x$ eigenvalues by an order of magnitude across the whole spectrum. Given that $\mu$ controls the amplitude of the noise term for the $x$ SDE (Eq. 37), this makes sense. As for the $y$ variable, the spectrum exhibits a small but discernible dependence on $\gamma$, with more prominent dependence on $(\mu, \gamma)$. We find that increasing $\mu$ tends to increase the amplitude of the higher energy eigenmodes (low eigenvalue number), with little impact on the low energy (high number) modes, thus preferentially elevating the left-part of the spectrum, and potentially eliminating the plateau depending on $\sigma$. In contrast, increasing $\sigma$ tends to generally increase the eigenmode amplitude across the spectrum, more preferentially for lower energy modes up to the flattening of the high energy mode spectral decay behavior at low eigenvalue number, resulting in a flat plateau for the whole region of eigenvalue numbers [1,100], for high $\sigma$ and low $\mu$. The fact that $\sigma$ has a significant impact on the fast variable dynamics is not surprising, given that it controls the amplitude of the noise in the $y$-SDE (Eq. 38). The similarly dominant role for $\mu$ reflects the slaving of the fast $y$-dynamics to the slow $x$-dynamics. In fact, we find the largest impact of increasing $\mu$ on $y$-dynamics when $\mu>>\sigma$, resulting in a $y$-eigenspectrum that is similar in structure to the $x$-spectrum, with no plateau, reflecting the dominance of the slow noise in determining the $y$-dynamics when the fast noise is negligible.

Next, comparing the spectra for the two schemes, in Fig. 8, we see first that there is similar spectral decay in the eigenvalue amplitudes for the top 5 dominant eigenmodes for both stiff and SCSP methods and for both $(x, y)$. The spectral decay rate, and agreement between the two schemes, continue over the range of all eigenvalues for $x$, with accelerating decay rate beyond the 200th eigenvalue. On the other hand, the decay rate of the $y$ eigenvalues from both schemes slows down after the 5th eigenmode, until accelerated decay is retrieved again beyond the 200th eigenvalue. Further, clearly, beyond the 10th eigenmode, significant differences are observed in the $y$-eigenvalue amplitudes available from the two schemes.

The first 5 eigenmodes are also illustrated, respectively, for the $x$ and $y$ variables, in Figures 9 and 10. The plots show the scaled eigenmodes, where the scale factor is the corresponding eigenvalue for each, to indicate the relative importance of different modes. We can see that, for both $x$ and $y$, the structure of the first five eigenmodes is essentially identical for the two schemes. The statistics for $x$, as well as the resulting agreement between the two schemes, is better than is found for $y$. In fact, significant disagreement in the spatial structure begins with the fifth mode, at $t \sim 0.5$, for $y$. Nonetheless, clearly the dominant eigenmodes of the autocovariance in both $x$ and $y$ are essentially equivalent between the two schemes.

We also show the PDFs of $x$ and $y$, at different time instances, from each of the 


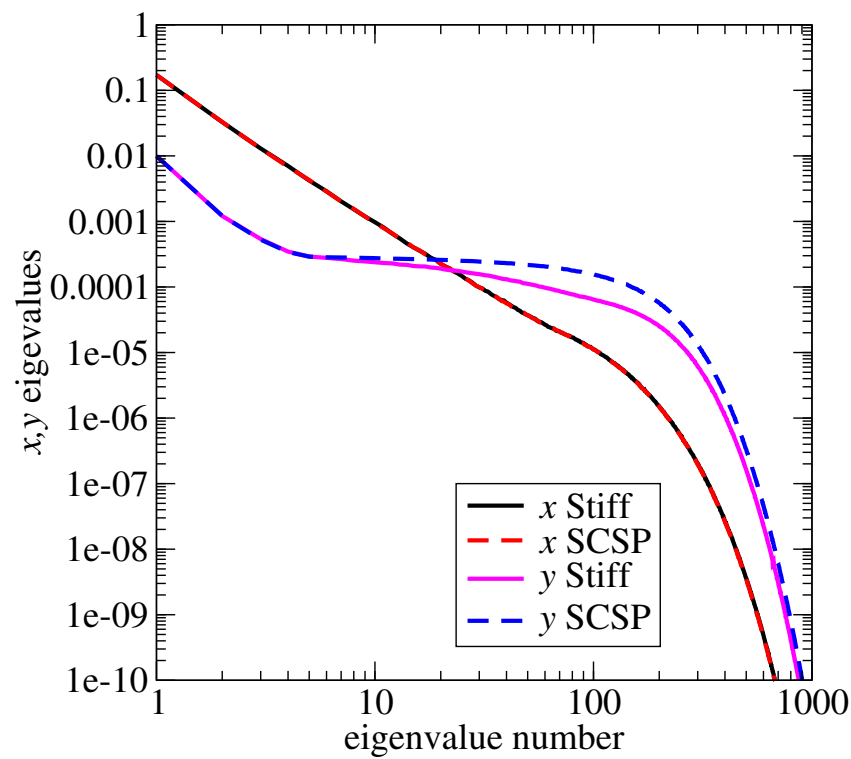

Figure 8: Decay of eigenvalue amplitudes for the stiff and SCSP-no-refinement cases. Employing $10^{4}$ samples, with the baseline conditions.

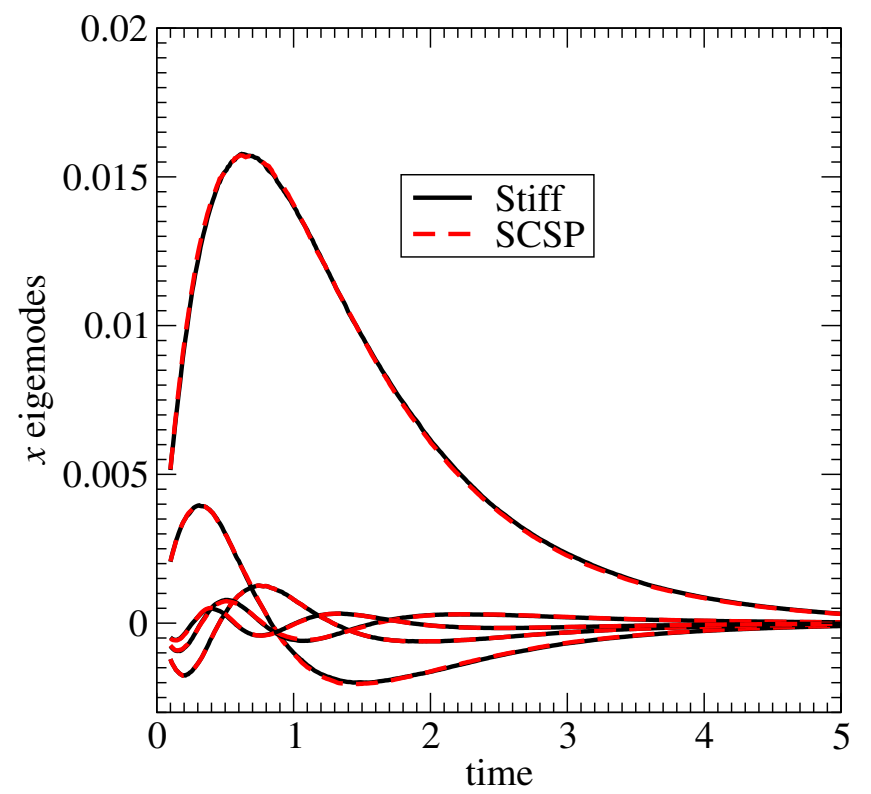

Figure 9: First 5 scaled eigenmodes of $x$ for the Stiff and SCSP-no-refinement cases. Employing $10^{4}$ samples, with the baseline conditions. 


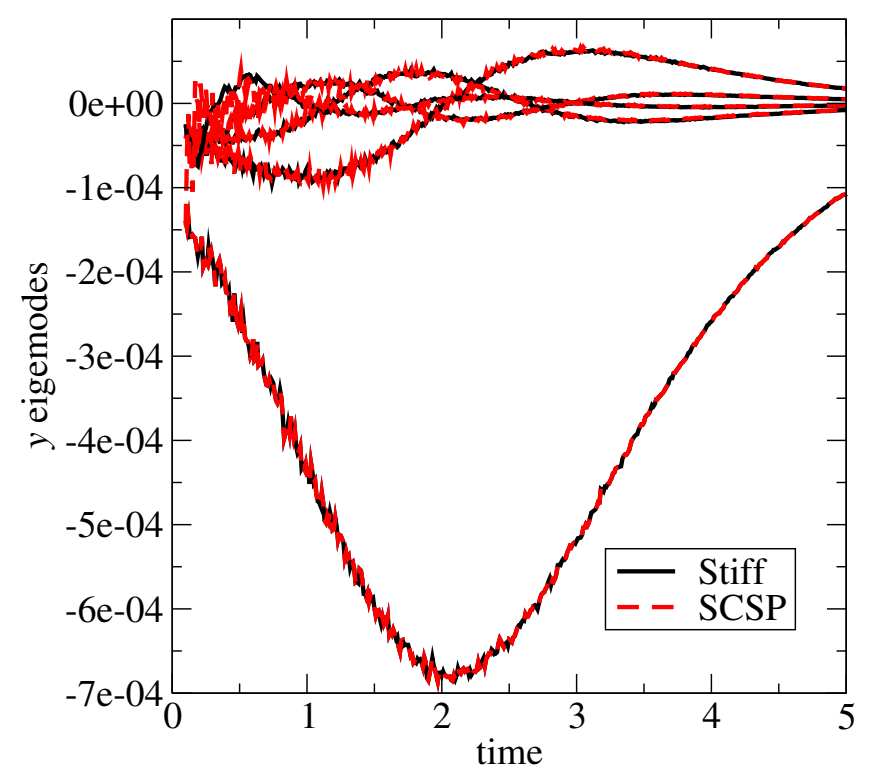

Figure 10: First 5 scaled eigenmodes of $y$ for the Stiff and SCSP-no-refinement cases. Employing $10^{4}$ samples, with the baseline conditions.

two schemes, in Figures 11 and 12. Here again we see generally similar structure between the two schemes, and with better agreement in $p(x)$, rather than $p(y)$. Further, the differences in $p(y)$ are highest at early time, consistent with the standard deviation comparison above.

Finally, we look at the convergence of the error in the SCSP solution, with time step size, in Figures 13 and Figures 14. These restuls are based on computed values of means and standard deviations of both variables, $x$ and $y$, at a given time instant, $t=1.28$. These statistics are computed based on $10^{5}$ sampled solution trajectories for a geometric sequence of time steps, $h_{\mathrm{SCSP}}=\{0.01,0.02, \cdots, 0.32\}$. Further, the errors in the computed statistics are averaged over 10 replicas. The self convergence results, in Fig. 13, are based on the error between statistics computed from SCSP solutions with consecutive time steps $(\Delta t, 2 \Delta t)$, while convergence results with respect to the stiff solution, in Fig. 14, are based on the replica-average error between the SCSP solution at any given time step, and reference statistics from $10^{6}$ samples of the stiff integrated solution with small time step $h_{\text {stiff }}=1.25 \cdot 10^{-4}$. Further, except for the $y$-stdv results, there is no discernible difference in these plots for the SCSP cases with 0 or 1 refinements. Thus, we only show the $y$-stdv 1-refinement results on both figures, as indicated. The results illustrate the first-order self-convergence of SCSP for both the fast and slow variables, and for both means and standard deviations, in Fig. 13, for both 0/1-refinement cases. The cross-convergence results relative to the fine time-step stiff results, in Fig. 14, show similar results for both $(x, y)$ means, and for the $x$ standard deviation. However, and already hinted at above, we see that the SCSP $y$-stdv results are clearly non-convergent, irrespective of refinement, relative to the stiff solver results. 


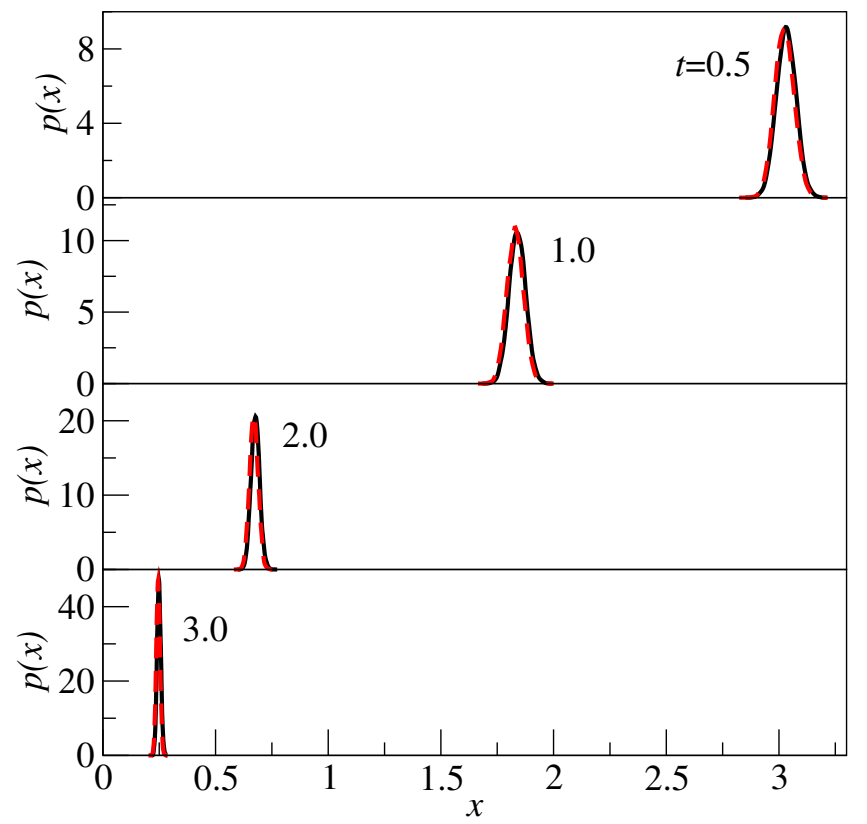

Figure 11: $x$-PDFs for the Stiff (black) and SCSP-no-refinement (red) cases. Employing $10^{4}$ samples, with the baseline conditions.

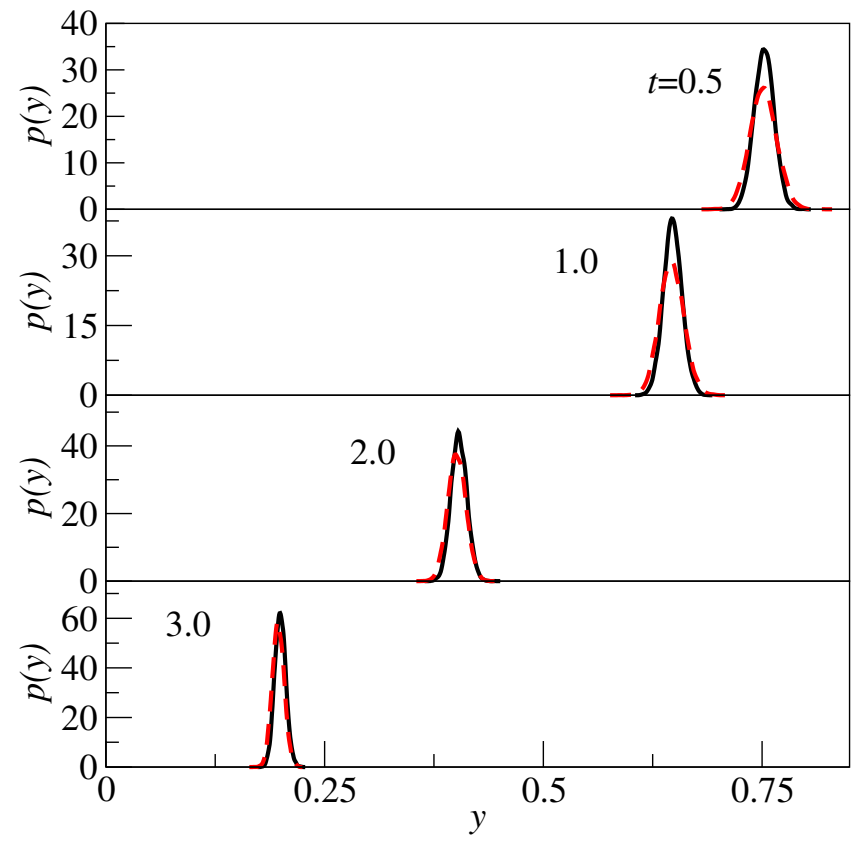

Figure 12: $y$-PDFs for the Stiff (black) and SCSP-no-refinement (red) cases. Employing $10^{4}$ samples, with the baseline conditions. 
Here, again, we point out that this is, effectively, by construction, given the inherent approximation of the fast dynamics by SCSP, in order to attain speedup, with large time-step explicit time integration of the SDE.

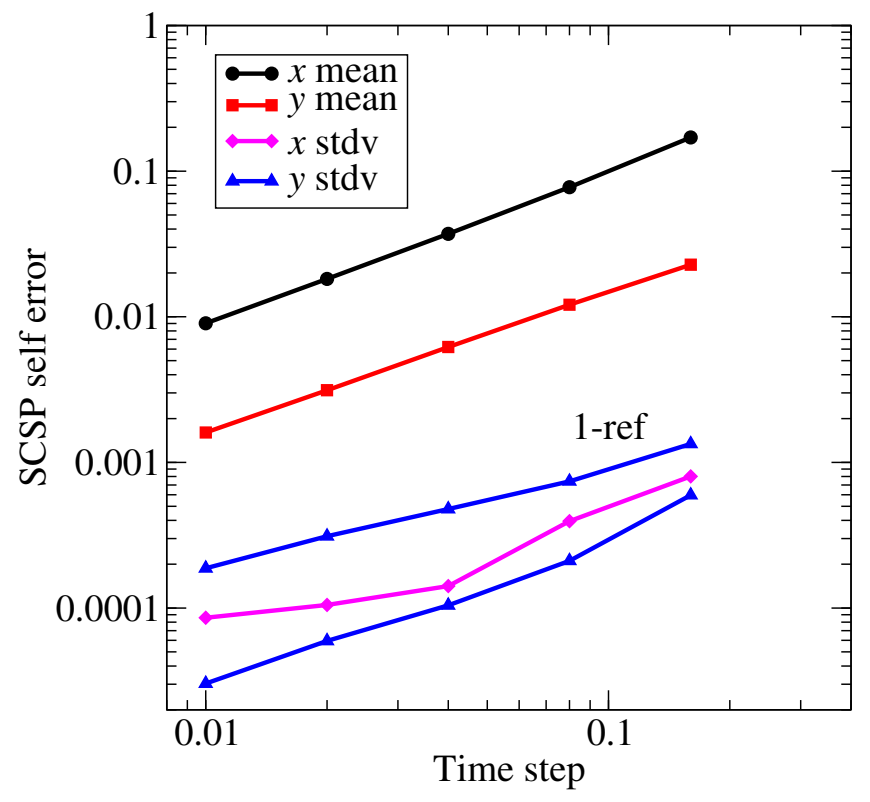

Figure 13: SCSP self convergence, employing 10-member ensembles of $10^{5}$ samples, with the baseline conditions, over a range of $h_{\mathrm{SCSP}}$. Unlabeled lines are all for no-refinement, while the $y$-stdv line labeled "1-ref" is for the case with one refinement. For the other results plotted, the data with one refinement are on top of the no-refinement data.

\section{Closing remarks}

Computational singular perturbation (CSP) is a powerful method for the analysis of deterministic dynamical systems with stiffness. It provides means for identification of fast and slow dynamical subspaces, and associated slow manifolds defined by the equilibration of fast processes. It has been used both for analysis of computed dynamics and for the construction of operator-split explicit time integration of stiff ordinary differential equation systems. However, CSP is not directly applicable to stochastic dynamical systems such as chemical reaction systems at micro or meso-scale. The key contribution of this work is the construction of a stochastic computational singular perturbation (SCSP) algorithm, by extending CSP to the stochastic context. This extension is non-trivial, due to the difficulty of decoupling fast and slow modes of both the drift and the volatility at the same time. We develop a SCSP scheme that allows the iterative construction of two sets of basis vectors spanning the fast and slow dynamical subspaces. With this construction, the fast and slow dynamics of a stiff stochastic differential equation (SDE) can be decoupled, while projecting onto the tensor product of the associated vector spaces. 


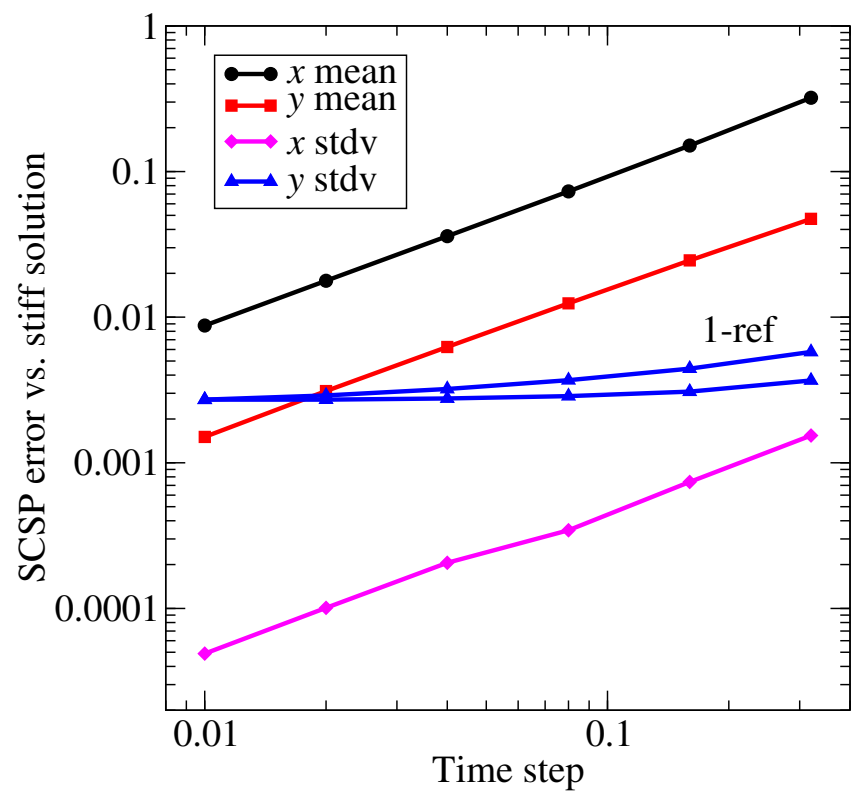

Figure 14: SCSP convergence with respect to the small- $\Delta t$ stiff solution, employing 10-member ensembles of $10^{5}$ samples, with the baseline conditions, over a range of $h_{\mathrm{SCSP}}$. Unlabeled lines are all for no-refinement, while the $y$-stdv line labeled " 1 -ref" is for the case with one refinement. For the other results plotted, the data with one refinement are on top of the no-refinement data. 
We developed an operator-split explicit time integration algorithm for solving stiff SDEs using SCSP. The construction follows the structure of the CSP time integrator for stiff ODEs. With decoupled fast and slow dynamics, the numerical simulation of a stiff SDE can be done by first approximating the fast dynamics by a stochastic algebraic relation, then integrating the system according to the slow dynamics. Since the stochastic algebraic relation describing the fast dynamics does not depend on the step length of time integration, and the subsequent time integration of the slow processes is non-stiff, the construction allows stable integration with large explicit time steps. Numerical experiments highlight the accuracy of computed first order statistics of the state variables, and that of the second order statistics of the slow variables, relative to stiff time integration of the original SDE system.

There is much more to be investigated in further detail, such as developing a more general formulation of SCSP that includes multidimensional noise, and constructing a rigorous description of the stochastic slow manifold. Further, while integration methods for stochastic partial differential equations (SPDEs), including stiffness, have attracted much attention in recent years (see, e.g., [32, 68, 69]), numerical construction of invariant manifolds for stiff SPDEs has not been tackled to date. When there is a clear separation of time scales, SCSP could potentially be extended to the analysis and integration of stiff SPDEs. Existing work on the application of CSP to analysis of deterministic PDE dynamics may be of use in this regard [25]. We hope that the present work, outlining elements of an algorithmic framework for analysis and simulation of stochastic chemical systems, can be further applied to a broad range of stiff stochastic systems in the applied sciences.

\section{Reference}

[1] L. Arnold. Random dynamical systems. Springer-Verlag, Berlin, 1998.

[2] Peter Benner and Tobias Damm. Lyapunov equations, energy functionals, and model order reduction of bilinear and stochastic systems. SIAM J. Control Optim., 49(2):686-711, 2011.

[3] Peter Benner and Martin Redmann. Model reduction for stochastic systems. Stochastic Partial Differential Equations: Analysis and Computations, 3(3):291338, 2015.

[4] N. Berglund and B. Gentz. Geometric singular perturbation theory for stochastic differential equations. Journal of Differential Equations, 191(1):1 - 54, Jun 2003.

[5] N. Berglund and B. Gentz. Noise-induced phenomena in slow-fast dynamical systems. Springer, London, 2006.

[6] M. Bodenstein and H. Lutkemeyer. Die photochemische bildung von bromwasserstoff und die bildungsgeschwindigkeit der brommolekul aus den atomen. Z. Phys. Chem., 114:208-236, 1924.

[7] E.M. Bulewicz, C.G. James, and T.M. Sugden. Photometric investigations of alkali metals in hydrogen flame gases, ii. Proc. Roy. Soc. (London), Series A, 235:89-106, 1956. 
[8] Yang Cao, Daniel T. Gillespie, and Linda R. Petzold. The slow-scale stochastic simulation algorithm. The Journal of Chemical Physics, 122(1), 2005.

[9] Tomás Caraballo, Jinqiao Duan, Kening Lu, and Björn Schmalfuß. Invariant manifolds for random and stochastic partial differential equations. Adv. Nonlinear Stud., 10(1):23-52, 2010.

[10] Mihai Cucuringu and Radek Erban. Adm-cle approach for detecting slow variables in continuous time markov chains and dynamic data. Technical report.

[11] M.J. Davis and R.T. Skodje. Geometric investigation of low-dimensional manifolds in systems approaching equilibrium. J. Chem. Phys., 111:859-874, 1999.

[12] B.J. Debusschere, Y.M. Marzouk, H.N. Najm, B. Rhoads, D.A. Goussis, and M. Valorani. Computational singular perturbation with non-parametric tabulation of slow manifolds for time integration of stiff chemical kinetics. Combustion Theory and Modelling, 16(1):173-198, 2012.

[13] Jinqiao Duan, Kening Lu, and Björn Schmalfuss. Invariant manifolds for stochastic partial differential equations. Ann. Probab., 31(4):2109-2135, 2003.

[14] Jinqiao Duan, Kening Lu, and Björn Schmalfuss. Smooth stable and unstable manifolds for stochastic evolutionary equations. J. Dynam. Differential Equations, 16(4):949-972, 2004.

[15] E.Hesstvedt, O.Hov, and I.S.A.Isaksen. Quasi-steady-state approximations in air pollution modeling: Comparison of two numerical schemes for oxidant prediction. Int. J. Chem. Kinetics, 10:971-994, 1978.

[16] A. Fehrst. Enzyme structure and mechnisms, 2nd Edition. Freeman, New York, 1975.

[17] D. T. Gillespie. The chemical langevin equation. J. Chem. Phys., 113(1):297 306, 2000.

[18] M.V. Tretyakov G.N. Milstein. Stochastic numerics for mathematical physics. Springer-Verlag Berlin Heidelberg, 2004.

[19] D.A. Goussis. On the Construction and Use of Reduced Chemical Kinetics Mechanisms Produced on the Basis of Given Algebraic Relations. J. Comput. Physics, 128:261-273, 1996.

[20] D.A. Goussis. Quasi steady state and partial equilibrium approximations: Their relation and their validity. Combustion Theory and Modelling, 16(5):869-926, 2012.

[21] D.A. Goussis and S.H. Lam. On the homogeneous methane-air reaction system. Report 1892-MAE, Princeton Univ., 1990.

[22] D.A. Goussis and S.H. Lam. A study of homogeneous methanol oxidation kinetic using csp. Proc. Comb. Inst., 24:113-120, 1992. 
[23] D.A. Goussis and H.N. Najm. Model Reduction and Physical Understanding of Slowly Oscillating Processes: The Circadian Cycle. Multiscale Modeling and Simulation, 5(4):1297-1332, 2006.

[24] D.A. Goussis and M. Valorani. An Efficient Iterative Algorithm for the Approximation of the Fast and Slow Dynamics of Stiff Systems. J. Comp. Phys., 214:316346, 2006.

[25] DA Goussis, M Valorani, F Creta, and HN Najm. Reactive and reactive-diffusive time scales in stiff reaction-diffusion systems. Progress in Computational Fluid Dynamics, 5(6):316-326, 2005.

[26] John Goutsias. Quasiequilibrium approximation of fast reaction kinetics in stochastic biochemical systems. The Journal of Chemical Physics, 122(18), 2005.

[27] Serkan Gugercin and Athanasios C. Antoulas. A survey of model reduction by balanced truncation and some new results. Internat. J. Control, 77(8):748-766, 2004.

[28] M. Hadjinicolaou and D. A. Goussis. Asymptotic solutions of stiff pdes with the csp method: the reaction-diffusion equation. SIAM J. Sci. Comput., 20:781-810, 1999.

[29] X. Han and H. Najm. Dynamical structures in stochastic chemical reaction systems. SIAM J. Applied Dynamical Systems, 13:1033 - 1351, 2014.

[30] J. A. M. Janssen. The elimination of fast variables in complex chemical reactions. II. Mesoscopic level (reducible case). J. Statist. Phys., 57(1-2):171-185, 1989.

[31] J. A. M. Janssen. The elimination of fast variables in complex chemical reactions. III. Mesoscopic level (irreducible case). J. Statist. Phys., 57(1-2):187-198, 1989.

[32] A. Jentzen and P. E. Kloeden. The numerical approximation of stochastic partial differential equations. Milan Journal of Mathematics, 77(1):205-244, 2009.

[33] A. Kazakov, M. Chaos, Z. Zhao, and F.L. Dryer. Computational Singular Perturbation Analysis of Two-Stage Ignition of Large Hydrocarbons. J. Phys. Chem. A, 110:7003-7009, 2006.

[34] J.C. Keck. Rate-controlled constrained-equilibrium theory of chemical reactions in complex systems. Prog. Energy Combust. Sci., 16:125-154, 1990.

[35] A. L. Kuharsky and A. L. Fogelson. Surface-mediated control of bood coagulation: the role of binding site densitites and platelet deposition. Biophysical Journal, 80:1050-1074, 2001.

[36] S. H. Lam. Using csp to understand complex chemical kinetics. Combust. Sci. Technol., pages 375-404, 1993.

[37] S. H. Lam and D. A. goussis. The csp mehtod for simplifying kinetics. Int. J. Chem. Kinet, 26:461-486, 1994. 
[38] S.H. Lam and D.A. Goussis. Understanding complex chemical kinetics with computational singular perturbation. Proc. Comb. Inst., 22:931-941, 1988.

[39] R. Law, M. Metgalchi, and J.C. Keck. Rate-controlled constrained equilibrium calculation of ignition delay times in hydrogen-oxygen mixtures. In Twenty-Second Symposium (International) on Combustion, pages 1705-1713. The Combustion Institute, 1988.

[40] J.C. Lee, H.N. Najm, S. Lefantzi, J. Ray, M. Frenklach, M. Valorani, and D. Goussis. A CSP and Tabulation Based Adaptive Chemistry Model. Combustion Theory and Modeling, 11(1):73-102, February 2007.

[41] T. Løvås, P. Amnéus, F. Mauss, and E. Mastorakos. Comparison of automatic reduction procedures for ignition chemistry. Proc. Comb. Inst., 29:1387-1393, 2002.

[42] Kening Lu and Björn Schmalfuß. Invariant manifolds for stochastic wave equations. J. Differential Equations, 236(2):460-492, 2007.

[43] Kening Lu and Björn Schmalfuß. Invariant foliations for stochastic partial differential equations. Stoch. Dyn., 8(3):505-518, 2008.

[44] T. Lu and C.K. Law. A directed relation graph method for mechanism reduction. Proc. Comb. Inst., 30:1333-1341, 2005.

[45] T. Lu and C.K. Law. Linear-time reduction of large kinetic mechanisms with directed relation graph: n-heptane and iso-octane. Combustion and Flame, 2005. in press.

[46] T. F. Lu, Y. G. Ju, and C. K. Law. complex csp for chemistry reduction and analysis. Combust. Flame, 126:1445-1455, 2001.

[47] U. Maas, R. W. Dibble, J. Wanatz, and E. Zwicker. Combustion: Physical and chemical fundamentals, modeling and simulation, experiment, pollutant formation, 2nd Edition. Springer, Berlin, 1999.

[48] U. Maas and S. B. Pope. Simplifying chemical kinetics: intrinsic lowdimensional manifolds in composition space. Combust. Flame, 88:239-264, 1992.

[49] A. Massias, D. diamantis, E. Mastorakos, and D. Goussis. Global reduced mechanisms for methane and hydrogen combustion with nitric oxide formation constracted with csp data. Combust. Theory Model, 3:233-257, 1999.

[50] H.N. Najm, D. Ponganis, and J. Prager. Analysis of NO Structure in a MethaneAir Edge Flame. Proc. Comb. Inst., 32(1):1117-1124, 2008.

[51] H.N. Najm, M. Valorani, D.A. Goussis, and J. Prager. Analysis of Methane-Air Edge Flame Structure. Combustion Theory and Modelling, 14(2):257-294, 2010. 
[52] M.S. Okino and M.L. Mavrovouniotis. Simplification of mathematical models of chemical kinetics. Chemical Reviews, 98:391-408, 1998.

[53] E. Platen P.E. Kloeden. Numerical solution of stochastic differential equations. Springer-Verlag Berlin Heidelberg, 1992.

[54] N. Peters and B. Rogg. Reduced kinetic mechanisms for applications in combustion systems. Springer, Berlin, 1993.

[55] J. Prager, H. Najm, M. Valorani, and D. Goussis. Skeletal Mechanism Generation with CSP and Validation for Premixed n-Heptane Flames. Proc. Comb. Inst., 32(1):509-517, 2009.

[56] J. Prager, H.N. Najm, M. Valorani, and D. Goussis. Structure of n-Heptane/Air Triple Flames in Partially-Premixed Mixing Layers. Combustion and Flame, 158:2128-2144, 2011.

[57] Christopher V. Rao and Adam P. Arkin. Stochastic chemical kinetics and the quasi-steady-state assumption: Application to the gillespie algorithm. The Journal of Chemical Physics, 118(11), 2003.

[58] Z. Ren, S.B. Pope, A. Vladimirsky, and J.M. Guckenheimer. The invariant constrained equilibrium edge preimage curve method for the dimension reduction of chemical kinetics. J. Chem. Phys., 124:114111, 2006.

[59] Howard Salis and Yiannis N. Kaznessis. An equation-free probabilistic steadystate approximation: Dynamic application to the stochastic simulation of biochemical reaction networks. The Journal of Chemical Physics, 123(21), 2005.

[60] B. Schmalfuss and K. R. Schneider. Existence of a slow invariant manifold for a class of random differential equations with two time scales. In EQUADIFF 2003, pages 919-921. World Sci. Publ., Hackensack, NJ, 2005.

[61] Björn Schmalfuss. Inertial manifolds for random differential equations. In Probability and partial differential equations in modern applied mathematics, volume 140 of IMA Vol. Math. Appl., pages 213-236. Springer, New York, 2005.

[62] Björn Schmalfuss and Klaus R. Schneider. Invariant manifolds for random dynamical systems with slow and fast variables. J. Dynam. Differential Equations, 20(1):133-164, 2008.

[63] L. A. Segel and M. Slemrod. The quasi-steady-state assumption: a case study in perturbation. SIAM Review, 31:446-477, 1989.

[64] J. H. Seinfeld and S. N. Pandis. Atmospheric chemistry and physics: from air pollution to climate change. Wiley, New York, 1998.

[65] Tatsuo Shibata. Reducing the master equations for noisy chemical reactions. The Journal of Chemical Physics, 119(13), 2003. 
[66] S. Singh, J. M. Powers, and S. Paolucci. On slow manifolds of chemically reactive systems. 117:1482-1697, 2002.

[67] Stephen Smith, Claudia Cianci, and Ramon Grima. Model reduction for stochatic chemical systems with abundant species. J. Chem. Phys., page 214105, 2015.

[68] Robert Strehl and Silvana Ilie. Hybrid stochastic simulation of reaction-diffusion systems with slow and fast dynamics. The Journal of Chemical Physics, 143(23):234108, 2015.

[69] Catherine Ta, Dongyong Wang, and Qing Nie. An integration factor method for stochastic and stiff reactiondiffusion systems. Journal of Computational Physics, 295:505 - 522, 2015.

[70] T. Turanyi, A. S. Tomlin, and M. J. Pilling. On the error of the quasi-steady-state approximation. J. Phys. Chem., 97:163-172, 1993.

[71] M. Valorani, F. Creta, F. Donato, H.N. Najm, and D.A. Goussis. Skeletal Mechanism Generation and Analysis for $n$-heptane with CSP. Proc. Comb. Inst., 31:483-490, 2007.

[72] M. Valorani, F. Creta, D.A. Goussis, J.C. Lee, and H.N. Najm. Chemical Kinetics Simplification via CSP. Combustion and Flame, 146:29-51, 2006.

[73] M. Valorani and D.A. Goussis. Explicit Time-Scale Splitting Algorithm For Stiff Problems: Auto-Ignition Of Gaseous-Mixtures Behind A Steady Shock. J. Comput. Phys., 169:44-79, 2001.

[74] M. Valorani, D.A. Goussis, F. Creta, and H.N. Najm. Higher Order Corrections in the Approximation of Low Dimensional Manifolds and the Construction of Simplified Problems with the CSP Method. J. Comput. Phys., 209:754-786, 2005.

[75] M. Valorani, H.N. Najm, and D. Goussis. CSP Analysis of a Transient FlameVortex Interaction: Time Scales and Manifolds. Combustion and Flame, 134(12):35-53, 2003.

[76] M. Valorani and S. Paolucci. The G-Scheme: A framework for multi-scale adaptive model reduction. Journal of Computational Physics, 228:4665-4701, 2009.

[77] E. I. Verriest. Time Variant Balancing and Nonlinear Balanced Realizations, pages 213-250. Springer Berlin Heidelberg, Berlin, Heidelberg, 2008.

[78] F. A. Williams. Combustion theory: the fundamental theory of chemically reacting flow systems, 2nd Edition. Benjamin/Cummings, Menlo Park, CA, 1985.

[79] F.A. Williams. Combustion Theory. Addison-Wesley, New York, 2nd edition, 1985.

[80] Fuke Wu, Tianhai Tian, James B. Rawlings, and George Yin. Approximate method for stochastic chemical kinetics with two-time scales by chemical langevin equations. The Journal of Chemical Physics, 144(17), 2016. 
[81] A. N. Yannacopoulos, A. S. Tomlin, J. Brindley, J. H. Merkin, and M. J. Pilling. The error of the quasi-steady-state approximation in spatially distributed systems. Chem. Phys. Lett., 248:63-70, 1996.

[82] Liqian Zhang, Biao Huang, and Tongwen Chen. Model reduction of uncertain systems with multiplicative noise based on balancing. SIAM J. Control Optim., 45(5):1541-1560 (electronic), 2006.

\section{Appendix}

\section{Evolution matrices in 3.1}

Using Jacobians in (39) and the basis vectors (40) and (41), the evolution of $f_{(0)}^{r}$, $g_{(0)}^{r}, f_{(0)}^{s}$ and $g_{(0)}^{s}$ in (42) - (45) follows (14) with

$$
\begin{array}{rlrl}
\Lambda_{0}^{r r} & =-\frac{3}{2} \gamma-\frac{3}{4} \Delta(x)+\frac{1}{2}, & & V_{0}^{r r}=\frac{9}{4} \gamma+\frac{3}{4} \Delta(x)-\frac{1}{2}, \\
\Lambda_{0}^{r s}=\frac{3}{2} \gamma+\frac{9}{4} \Delta(x)-\frac{3}{2}, & V_{0}^{r s}=\frac{3}{2} \gamma+\frac{3}{2} \Delta(x)-1, \\
\Lambda_{0}^{s r}=-\frac{1}{2} \gamma-\frac{1}{4} \Delta(x)+\frac{1}{2}, & V_{0}^{s r}=\frac{3}{4} \gamma+\frac{1}{4} \Delta(x)-\frac{1}{2}, \\
\Lambda_{0}^{s s}=\frac{1}{2} \gamma+\frac{3}{4} \Delta(x)-\frac{3}{2}, & V_{0}^{s s}=\frac{1}{2} \gamma+\frac{1}{2} \Delta(x)-1, \\
\Gamma_{0}^{r r}=-\sigma \sqrt{\gamma}+\frac{1}{2} \mu, & T_{0}^{r r}=\frac{3}{2} \sigma \sqrt{\gamma}-\frac{1}{2} \mu, \\
\Gamma_{0}^{r s}=\sigma \sqrt{\gamma}-\frac{3}{2} \mu, & T_{0}^{r s}=\frac{1}{2} \sigma \sqrt{\gamma}-\frac{1}{2} \mu, \\
\Gamma_{0}^{s r}=\frac{1}{2} \sigma \sqrt{\gamma}-\frac{3}{4} \mu, & T_{0}^{s r}=-\frac{3}{2} \sigma \sqrt{\gamma}+\frac{3}{2} \mu, \\
\Gamma_{0}^{s s}=-\frac{1}{2} \sigma \sqrt{\gamma}+\frac{9}{4} \mu, & T_{0}^{s s}=-\frac{1}{2} \sigma \sqrt{\gamma}+\frac{3}{2} \mu . \\
\tilde{\Lambda}_{0}^{r r}= & -\frac{3}{2} \gamma-\frac{3}{4} \Delta(x)+\frac{1}{2}+\frac{\frac{3}{2} \gamma+\frac{9}{4} \Delta(x)-\frac{3}{2}}{-\frac{3}{2} \gamma-\frac{3}{4} \Delta(x)+\frac{1}{2}}\left(-\frac{1}{2} \gamma-\frac{1}{4} \Delta(x)+\frac{1}{2}\right), \\
\tilde{\Gamma}_{0}^{r r}= & -\sigma \sqrt{\gamma}+\frac{1}{2} \mu+\frac{\frac{3}{2} \gamma+\frac{3}{2} \Delta(x)-1}{\frac{9}{4} \gamma+\frac{3}{4} \Delta(x)-\frac{1}{2}}\left(\frac{1}{2} \sigma \sqrt{\gamma}-\frac{3}{4} \mu\right) .
\end{array}
$$

Simplified stochastic and deterministic fast amplitudes are

$$
\begin{aligned}
f_{(1)}^{r}= & \left(\frac{3}{4}+\frac{1}{4} \kappa_{1}(x)\right)\left(-\gamma y+\gamma \frac{x}{1+x}\right) \\
& -\frac{\left(2 x^{3}+6 x^{2}+3 x+5\right) x}{\gamma\left(6 x^{3}+18 x^{2}+21 x+9\right)-2 x^{3}-6 x^{2}-3 x-5}, \\
g_{(1)}^{r}= & \left(-\frac{1}{2}+\frac{1}{2} \kappa_{2}(x)\right) \sigma \sqrt{\gamma} y \\
& +\frac{\mu x}{2} \frac{9 \gamma x^{3}+27 \gamma x^{2}-10 x^{3}+42 \gamma x-30 x^{2}+24 \gamma-15 x-25}{9 \gamma x^{3}+27 \gamma x^{2}-2 x^{3}+30 \gamma x-6 x^{2}+12 \gamma-3 x-5} .
\end{aligned}
$$


Evolution matrices in 3.2

In addition, the evolution of $\ell_{0}=\left(f_{(0)}^{r}, f_{(0)}^{s}, g_{(0)}^{r}, g_{(0)}^{s}\right)^{T}$ in equations (52) - (55) follows (14) with

$$
\begin{array}{llll}
\Lambda_{0}^{r r}=-\gamma, & \Lambda_{0}^{r s}=0, & \Lambda_{0}^{s r}=0, & \Lambda_{0}^{s s}=-1, \\
V_{0}^{r r}=-\gamma, & V_{0}^{r s}=\frac{\gamma}{\gamma-1} \Delta(x), & V_{0}^{s r}=0, & V_{0}^{s s}=\frac{1}{1-\gamma}, \\
\Gamma_{0}^{r r}=\sigma \sqrt{\gamma}, & \Gamma_{0}^{r s}=\sigma \sqrt{\gamma} \Delta(x), & \Gamma_{0}^{s r}=0, & \Gamma_{0}^{s s}=\mu(\gamma-1), \\
T_{0}^{r r}=\sigma \sqrt{\gamma}, & T_{0}^{r s}=0, & T_{0}^{s r}=0, & T_{0}^{s s}=\mu .
\end{array}
$$

Refinements for eigenvectors

By using (16), the iterative formulation for $\boldsymbol{b}_{(k)}^{r}$ and $\boldsymbol{\beta}_{(k)}^{r}$ can be calculated to be

$$
\begin{aligned}
\boldsymbol{b}_{(k)}^{r} & =\boldsymbol{b}_{(k-1)}^{r}+\left[\Lambda_{k-1}^{r r}\right]^{-1}\left[\Lambda_{k-1}^{r s}\right] \boldsymbol{b}_{(k-1)}^{s} \\
& =\boldsymbol{b}_{(k-2)}^{r}+\left[\Lambda_{k-2}^{r r}\right]^{-1}\left[\Lambda_{k-2}^{r s}\right] \boldsymbol{b}_{(k-2)}^{s}+\left[\Lambda_{k-1}^{r r}\right]^{-1}\left[\Lambda_{k-1}^{r s}\right] \boldsymbol{b}_{(k-1)}^{s} \\
& =\cdots \cdots \\
& =\boldsymbol{b}_{(0)}^{r}-\frac{1}{\gamma} \boldsymbol{b}_{(0)}^{s} \sum_{i=0}^{k-1} \Lambda_{i}^{r s}=\left(1-\frac{\Delta_{j}}{\gamma-1}-\frac{1}{\gamma(\gamma-1)} \sum_{i=0}^{k-1} \Lambda_{i}^{r s}\right), \\
\boldsymbol{\beta}_{(k)}^{r} & =\boldsymbol{\beta}_{(0)}^{r}-\frac{1}{\gamma} \boldsymbol{\beta}_{(0)}^{s} \sum_{i=0}^{k-1} V_{i}^{r s}=\left(1-\frac{1}{\gamma} \sum_{i=0}^{k-1} V_{i}^{r s}\right),
\end{aligned}
$$

and accordingly

$$
\begin{aligned}
& \boldsymbol{a}_{(k)}^{s}=\left[I_{2}-\boldsymbol{a}_{(0)}^{r} \boldsymbol{b}_{(k)}^{r}\right]\left[I_{2}-\boldsymbol{a}_{(0)}^{r} \boldsymbol{b}_{(k-1)}^{r}\right] \cdots\left[I_{2}-\boldsymbol{a}_{(0)}^{r} \boldsymbol{b}_{(1)}^{r}\right] \boldsymbol{a}_{(0)}^{s}, \\
& \boldsymbol{\alpha}_{(k)}^{s}=\left[I_{2}-\boldsymbol{\alpha}_{(0)}^{r} \boldsymbol{\beta}_{(k)}^{r}\right]\left[I_{2}-\boldsymbol{\alpha}_{(0)}^{r} \boldsymbol{\beta}_{(k-1)}^{r}\right] \cdots\left[I_{2}-\boldsymbol{\alpha}_{(0)}^{r} \boldsymbol{\beta}_{(1)}^{r}\right] \boldsymbol{\alpha}_{(0)}^{s} .
\end{aligned}
$$

Therefore after $k(\geq 1)$ iteration of refinements the modes of vector fields become

$$
\begin{aligned}
& f_{(k)}^{r}=\boldsymbol{b}_{(k)}^{r} \boldsymbol{f}(\boldsymbol{z})=-\gamma y+\gamma \frac{x}{1+x}-\frac{x}{(1+x)^{2}}+x\left(\frac{\Delta_{j}}{\gamma-1}+\frac{\sum_{i=0}^{k-1} \Lambda_{i}^{r s}}{\gamma(\gamma-1)}\right), \\
& g_{(k)}^{r}=\boldsymbol{\beta}_{(k)}^{r} \boldsymbol{g}(\boldsymbol{z})=\sigma \sqrt{\gamma} y-\frac{\mu x}{\gamma} \sum_{i=0}^{k-1} V_{i}^{r s}, \\
& f_{(k)}^{s}=\boldsymbol{b}_{(k)}^{s} \boldsymbol{f}(\boldsymbol{z})=-\frac{x}{\gamma-1}, \\
& g_{(k)}^{s}=\boldsymbol{\beta}_{(k)}^{s} \boldsymbol{g}(\boldsymbol{z})=\mu x .
\end{aligned}
$$

By using (66) - (69), after the first step of refinement we have

$$
\begin{aligned}
& \boldsymbol{b}_{(1)}^{r}=\boldsymbol{b}_{(0)}^{r}-\frac{1}{\gamma} \Lambda_{0}^{r s} \boldsymbol{b}_{(0)}^{s}=\left(1-\frac{\Delta(x)}{\gamma}-\frac{\Delta_{j}}{\gamma(\gamma-1)}\right), \\
& \boldsymbol{\beta}_{(1)}^{r}=\boldsymbol{\beta}_{(0)}^{r}-\frac{1}{\gamma} V_{0}^{r s} \boldsymbol{\beta}_{(0)}^{s}=\left(1-\frac{\Delta(x)}{\gamma}-\frac{\Delta_{j}}{\gamma(\gamma-1)}\right),
\end{aligned}
$$


and accordingly

$$
\begin{aligned}
& \boldsymbol{a}_{(1)}^{s}=\left[I_{2}-\boldsymbol{a}_{(0)}^{r} \boldsymbol{b}_{(1)}^{r}\right] \boldsymbol{a}_{(0)}^{s}=\left(\begin{array}{c}
\frac{\Delta(x)(\gamma-1)}{\gamma}+\frac{\Delta_{j}}{\gamma} \\
\gamma-1
\end{array}\right), \\
& \boldsymbol{\alpha}_{(1)}^{s}=\left[I_{2}-\boldsymbol{\alpha}_{(0)}^{r} \boldsymbol{\beta}_{(1)}^{r}\right] \boldsymbol{\alpha}_{(0)}^{s}=\left(\begin{array}{c}
\frac{\Delta(x)}{\gamma}+\frac{\Delta_{j}}{\gamma(\gamma-1)} \\
1
\end{array}\right) .
\end{aligned}
$$

Further, according to (70)-(73), we have

$$
\begin{aligned}
& f_{(1)}^{r}=-\gamma y+\gamma \frac{x}{1+x}-\frac{x}{(1+x)^{2}}+\frac{\Delta_{j}}{\gamma(\gamma-1)} x+\frac{\Delta(x)}{\gamma} x, \\
& g_{(1)}^{r}=\sigma \sqrt{\gamma} y-\frac{\mu x}{\gamma}\left(\Delta(x)+\frac{\Delta_{j}}{\gamma-1}\right), \\
& f_{(1)}^{s}=-\frac{x}{\gamma-1}, \\
& g_{(1)}^{s}=\mu x .
\end{aligned}
$$

Explicit stratonovich time integration for (37)-(38)

$$
\begin{aligned}
& x_{j+1}=x_{j}-x_{j} h+\frac{\mu \xi \sqrt{h}}{2}\left(x_{j}+x_{j+1}\right), \\
& y_{j+1}=y_{j}+\left(-\gamma y_{j}+\gamma \frac{x_{j}}{1+x_{j}}-\frac{x_{j}}{\left(1+x_{j}\right)^{2}}\right) h+\frac{\sigma \xi \sqrt{\gamma h}}{2}\left(y_{j}+y_{j+1}\right),
\end{aligned}
$$

or equivalently,

$$
\begin{aligned}
& x_{j+1}=\frac{2}{2-\mu \xi \sqrt{h}}\left(1-h+\frac{\mu \xi}{2} \sqrt{h}\right) x_{j}, \\
& y_{j+1}=\frac{2}{2-\sigma \xi \sqrt{\gamma h}}\left(\left(1-\gamma h+\frac{\sigma \xi \sqrt{\gamma h}}{2}\right) y_{j}+\frac{x_{j}}{1+x_{j}} \gamma h-\frac{x_{j}}{\left(1+x_{j}\right)^{2}} h\right) .
\end{aligned}
$$

